\title{
Magnetic neutron scattering studies on the Fe-based superconductor system $\mathrm{Fe}_{1+y} \mathrm{Te}_{1-x} \mathrm{Se}_{x}$
}

\author{
Jinsheng Wen* \\ Center for Superconducting Physics and Materials, National Laboratory of Solid State \\ Microstructures, Department of Physics, and Collaborative Innovation Center of Advanced \\ Microstructures, Nanjing University, Nanjing 210093, China
}

\begin{abstract}
I present a brief overview on the interplay between magnetism and superconductivity in one of the Fe-based superconductor systems, $\mathrm{Fe}_{1+y} \mathrm{Te}_{1-x} \mathrm{Se}_{x}$, where the research of our group has centered. The parent compound $\mathrm{Fe}_{1+y} \mathrm{Te}$ is an antiferromagnet; with Se doping, antiferromagnetic order is suppressed, followed by the appearance of superconductivity; optimal superconductivity is achieved when $x \sim 50 \%$, with a superconducting temperature $T_{c}$ of $\sim 15 \mathrm{~K}$. The parent compound has an in-plane magnetic ordering wave vector around $(0.5,0)$ (using the tetragonal notation with two Fe atoms per cell). When Se concentration increases, the spectral weight appears to be shifted to the wave vector around $(0.5,0.5)$, accompanying the optimization of superconductivity. A neutron-spin resonance has been observed around $(0.5,0.5)$ below $T_{c}$, and is suppressed, along with superconductivity, by an external magnetic field. Taking these evidences into account, it is concluded that magnetism and superconductivity in this system couple to each other closely — while the static magnetic order around $(0.5,0)$ competes with superconductivity, the spin excitations around $(0.5,0.5)$ may be an important ingredient for it. I will also discuss the nature of magnetism and substitution effects of $3 d$ transition metals.
\end{abstract}

Keywords: Fe-based superconductors, magnetic order, spin excitations

*jwen@nju.edu.cn 


\section{Introduction}

\subsection{A brief overview}

The quest for an alternative superconducting mechanism was soon initiated after the discovery of high-temperature superconductivity in the lamellar copper-oxide materials [1 3 ], which posed a great challenge to BCS theory [4], the many-body theory developed by Bardeen, Cooper, and Schrieffer that successfully explained conventional superconductivity [4]. The key concept is that electrons form pairs aided by the electron-phonon interactions, and the pairs condense at the superconducting temperature $T_{c}$. In the cuprate superconductors, electron-phonon coupling is not sufficient to induce superconductivity with such high $T_{c} \mathrm{~s}[5,6]$. In these materials, superconductivity develops from electronically doping a Mott insulator, and is in close proximity to the antiferromagnetic phase [5 9]. Thus, it is very promising that one may eventually work out the high- $T_{c}$ mechanism by studying the interplay between magnetism and superconductivity.

Research on this subject gained substantial momentum in 2008 with the discovery of superconductivity in compounds that contain Fe instead of $\mathrm{Cu}$ (termed "Fe-based superconductors") [10, 11]. The field was initially excited by the discovery of superconductivity in $\mathrm{LaFeAsO}_{1-x} \mathrm{~F}_{x}$ (labeled 1111 based on the elemental ratios in the chemical formula of the parent material) with $T_{c}=26 \mathrm{~K}$ by Hosono's group 11], following the group's earlier report of superconductivity in $\mathrm{LaFePO}_{1-x} \mathrm{~F}_{x}$ with $T_{c} \sim 5 \mathrm{~K}[10]$. Soon after the initial discovery, the scientific community witnessed a burst of new Fe-based superconductors. So far, besides the 1111 system, other five major families of Fe-based superconductors have been discovered, typified by $\mathrm{BaFe}_{2} \mathrm{As}_{2}$ (122) 12 15], LiFeAs (111) 16 22], $\mathrm{Fe}_{1+y} \mathrm{Te}_{1-x} \mathrm{Se}_{x}$ (11) 23-27], $\mathrm{Sr}_{2} \mathrm{VO}_{3} \mathrm{FeAs}$ (21311) [28, 29], and $A_{x} \mathrm{Fe}_{2-y} \mathrm{Se}_{2}(A=$ alkaline elements) $[30-34]$. The crystal structures for the five families are shown in Fig. 1 (The $A_{x} \mathrm{Fe}_{2-y} \mathrm{Se}_{2}$ compounds are isostructural to the 122) [10, 20, 35 38]. They are all tetragonal at room temperature, and have layered structures. The current record of $T_{c}$ in the bulk materials is $56 \mathrm{~K}$ 
in $\mathrm{Gd}_{1-x} \mathrm{Th}_{x} \mathrm{FeAsO}$ [39, 40]. This makes the Fe-based superconductors second only to cuprates in $T_{c}$, and for this reason they are often considered as another class of high-temperature superconductors. Comparing the differences and similarities between the two classes may help find the common ground underlying the high $T_{c}$ in these superconductors.

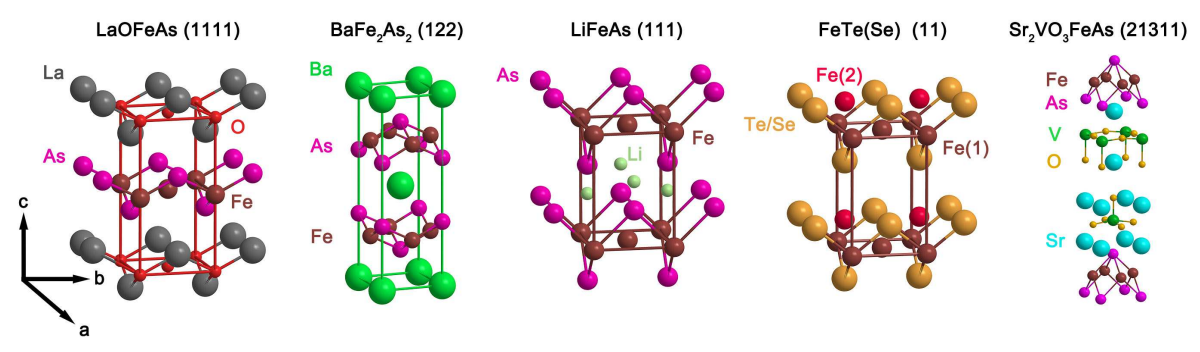

Figure 1: Schematic crystal structures for the 1111, 122, 111, 11, and 21311 type Fe-based superconductors.

The distinct properties of the parent compound set the Fe-based superconductors apart from the copper oxides. The undoped cuprates are Mott insulators, which are predicted by band theory to be metallic but turn out to be insulating because the otherwise itinerant electrons are localized due to the large Coulomb repulsion [5, 6]. In the Fe-based superconductors, their parent compounds are metallic [41]. This naturally leads to a different starting point, and a weak-coupling theory is often more favorable [42, 43]. Furthermore, unlike cuprates where only a single $\mathrm{Cu} 3 d$ band is involved, four or five Fe $3 d$ orbitals are involved in the multiple bands that cross the Fermi level [43]. This in some cases can complicate the interpretations [44]. Despite the differences, these two classes of high-temperature superconductors share surprisingly similar phase diagrams [45]. With very few exceptions in the Fe-based superconductors, the parent compounds exhibit long-range antiferromagnetic order, which is suppressed with doping, and superconductivity appears above a certain doping level 36 38, 46 64], resembling the phase diagrams of the cuprate superconductors [5-9]. Such a similarity immediately leads to the speculation that 
the pairing mechanism may be the same, with magnetic excitations replacing phonons in the electron pairing interactions [41 43, 65 68]. However, because of the multiband nature of the superconductivity, orbital excitations have also been proposed as possible contributors to the pairing mechanism [44, 69, 70]. Although a consensus on the high- $T_{c}$ mechanism has not been reached so far, it is generally believed that studying the magnetic correlations has and will continue to yield important results that are critical in understanding the high- $T_{c}$ superconductivity.

With the tremendous efforts on studying the Fe-based superconductors, there has been a plethora of literature on this topic. A great number of comprehensive and topical review articles are already available [45, 63, 71 [83]. In the current work, I will only focus on the $\mathrm{Fe}_{1+y} \mathrm{Te}_{1-x} \mathrm{Se}_{x}$ system, with emphasis on the neutron scattering studies of the magnetism within this system.

\section{2. $F e_{1+y} T e_{1-x} S e_{x}$ system}

Superconductivity in the $\mathrm{Fe}_{1+y} \mathrm{Te}_{1-x} \mathrm{Se}_{x}$ system was discovered in $\mathrm{Fe}_{1+y} \mathrm{Se}$, with zero resistance at $T \approx 8 \mathrm{~K}[23]$. For this system, the highest $T_{c}$ at ambient pressure was found to be $\sim 15 \mathrm{~K}$ for Se concentration around 0.5 [24, 25, 27]. Under pressure, the $T_{c}$ in $\mathrm{Fe}_{1+y} \mathrm{Se}$ could be raised up to $37 \mathrm{~K}$ [84 86$]$. A more significant enhancement of the $T_{c}$ was reported by scanning tunneling microscopy (STM) measurements on a single-layer FeSe thin film [87].It was shown that there was a possible superconducting gap of $20 \mathrm{meV}$, suggesting that the $T_{c}$ could be above $77 \mathrm{~K}$, although transport measurements only found zero resistance below $30 \mathrm{~K}$ [87]. Later angle-resolved-photoemission-spectroscopy (ARPES) measurements revealed a gap up to $20 \mathrm{meV}$ that closed at $\sim 65 \mathrm{~K}[88-$ 90]. More recently, transport measurements showed higher $T_{c}$ than that observed in the initial report [87, 91, 92]. In particular, superconducting-like electrical behavior was observed at temperatures above $100 \mathrm{~K}$ from the insitu transport measurements on the monolayer FeSe films using a four-probe method [91]. These studies suggested that $56 \mathrm{~K}$, the record of $T_{c}$ for the Febased superconductors might have been broken [39, 40, 93]. 
Aside from the possible high $T_{c}$ in thin films, there are several other features that highlight the importance of this system. For example: i) The magnetism in its parent compound may have a different origin than in the other Fe-based compounds, as will be discussed in Sec. 22 ii) As can be seen from Fig. 10 the crystal structure of the 11 system is the simplest; iii) As is obvious from the chemical formula, the 11 system does not contain As, so it is safer to handle; iv) Last but not least, large-size single crystals can be made available for this system (with $x \leq 0.7$ ). This is especially important for neutron scattering experiments because the limitations of neutron source strength and small scattering cross sections require the use of samples of considerable size $\left(\gtrsim 1 \mathrm{~cm}^{3}\right)$ to obtain reliable data in the given beamtime.

\subsection{Neutron scattering experiments}

Before I proceed, I shall discuss neutron scattering, the most powerful tool in characterizing magnetic correlations. Neutron scattering is analogous to Xray scattering [94]. They both can be used to probe the crystal structure and lattice dynamics (i.e. phonons), but since a neutron carries a spin of $1 / 2$, it can interact with the spin of the unpaired electron, allowing the material's magnetic information to be revealed. Neutrons are charge neutral and interact with matter through short-range weak forces, thus they have large penetration depth. This makes the bulk properties easily accessible by neutrons. The following discussions will be mostly on the magnetic scattering, whose cross section can be written as 95 - 97 ]

$$
\frac{d^{2} \sigma}{d \Omega d E_{f}}=\frac{N}{\hbar} \cdot \frac{k_{f}}{k_{i}} \cdot p^{2} \mathrm{e}^{-2 W} S(\mathbf{Q}, E),
$$

where $N$ is the total number of the unit cells, $k_{f}$ and $k_{i}$ are the final and incident

neutron wave vectors respectively, $p=\frac{\gamma r_{0}}{2} g f(\mathbf{Q}), \frac{\gamma r_{0}}{2}=0.2695 \times 10^{-12} \mathrm{~cm}, g$ is the Landé splitting factor, $f(\mathbf{Q})$ is the the wave vector $\mathbf{Q}$-dependent magnetic form factor, $\mathrm{e}^{-2 \mathrm{~W}}$ is the Debye-Waller factor, and $S(\mathbf{Q}, E)$ is the $\mathbf{Q}$ - and energy $E$-dependent dynamical spin correlation function, which is the quantity one would like to obtain eventually. $S(\mathbf{Q}, E)$ is related to the imaginary part 
of the dynamical spin susceptibility, $\chi^{\prime \prime}(\mathbf{Q}, E)$ via the fluctuation-dissipation theorem [98],

$$
S(\mathbf{Q}, E)=\frac{\hbar}{\pi g^{2} \mu_{\mathrm{B}}^{2}} \cdot \frac{1}{1-\mathrm{e}^{-E / \mathrm{k}_{\mathrm{B}} T}} \cdot \chi^{\prime \prime}(\mathbf{Q}, E),
$$

where $\mu_{\mathrm{B}}$ is the Bohr magneton, and $\left(1-\mathrm{e}^{-E / \mathrm{k}_{\mathrm{B}} T}\right)^{-1}$ is referred to as the Bose factor. It also satisfies a simple moment sum rule for a system with spins $S$ when one performs the integration over a Brillouin zone, $\int_{\mathrm{BZ}} S(\mathbf{Q}, E) d^{3} \mathbf{Q} d E=$ $S(S+1)$ [95, 96].

Triple-axis spectrometers (TAS) and time-of-flight spectrometers (TOFS) that can probe both elastic and inelastic scattering processes are commonly used. For a TAS, the three axes correspond to monochromator, sample, and analyzer axes. When doing an experiment with a TAS, one often fixes the final energy $E_{f}$ and varies the incident energy $E_{i}$. The energy is usually determined from the neutron wave length $\lambda$ by $E=81.79 / \lambda^{2}$, and $\lambda$ is determined using the reflection from a single-crystal plane according to the Bragg law $\lambda=2 d \sin \theta$. The spacing of the reflection plane $d$ is defined by $d=2 \pi / \sqrt{\frac{H^{2}}{a^{2}}+\frac{K^{2}}{b^{2}}+\frac{L^{2}}{c^{2}}}$, with $(H K L)$ being the Miller index, and $a, b, c$ being the lattice constants. The angle between the incident beam and the plane is $\theta$. Pyrographites with (002) plane are often used as the monochromator and analyzer. It is quite convenient to probe $S(\mathbf{Q}, E)$ at a specific $\mathbf{Q}-E$ point precisely utilizing a TAS, with good $\mathbf{Q}$ and $E$ resolution. It is suitable to measure systems where the signals are concentrated at certain regions. The disadvantage is that it only accesses a single point of the momentum-energy space at a time. If the spectral weight spreads over a wide range of $\mathbf{Q}$ and $E$, it will be very time consuming doing such an experiment. Also, the energy range is limited. For a TOFS, there is an array of position-sensitive detectors that allow a portion instead of a point of the momentum-energy space to be covered. The energies in this case are measured by the time neutrons taken to travel. Compared with the TAS, the availability of the TOFS is more limited, and the resolution is often more coarse for certain type of TOFS. Since they both have merits of their own, they are often used in complement to each other. 
In describing the neutron scattering data, the wave vector $\mathbf{Q}$ of $\left(\mathbf{Q}_{x}, \mathbf{Q}_{y}, \mathbf{Q}_{z}\right)$ expressed in $(H K L)$ has the reciprocal lattice unit (rlu) of $\left(a^{*}, b^{*}, c^{*}\right)=(2 \pi / a, 2 \pi / b, 2 \pi / c)$. Throughout this paper, the tetragonal notation with two Fe atoms per cell is used (as shown in Fig. (3). In this case the lattice constant $a \approx 3.8 \AA$.

\subsection{Organization of the article}

The remainder of this paper is organized as follows: in Sec. 2 results on the static magnetic order in $\mathrm{Fe}_{1+y} \mathrm{Te}_{1-x} \mathrm{Se}_{x}$ will be presented; in Sec. 3, the spin dynamics of this system will be shown; in Sec. 4, the origin of the magnetic excitations will be discussed briefly; in Sec. [5, the substitution effects using $3 d$ transition metals will be summarized, followed by the conclusions in Sec. 6.

\section{Static magnetic order}

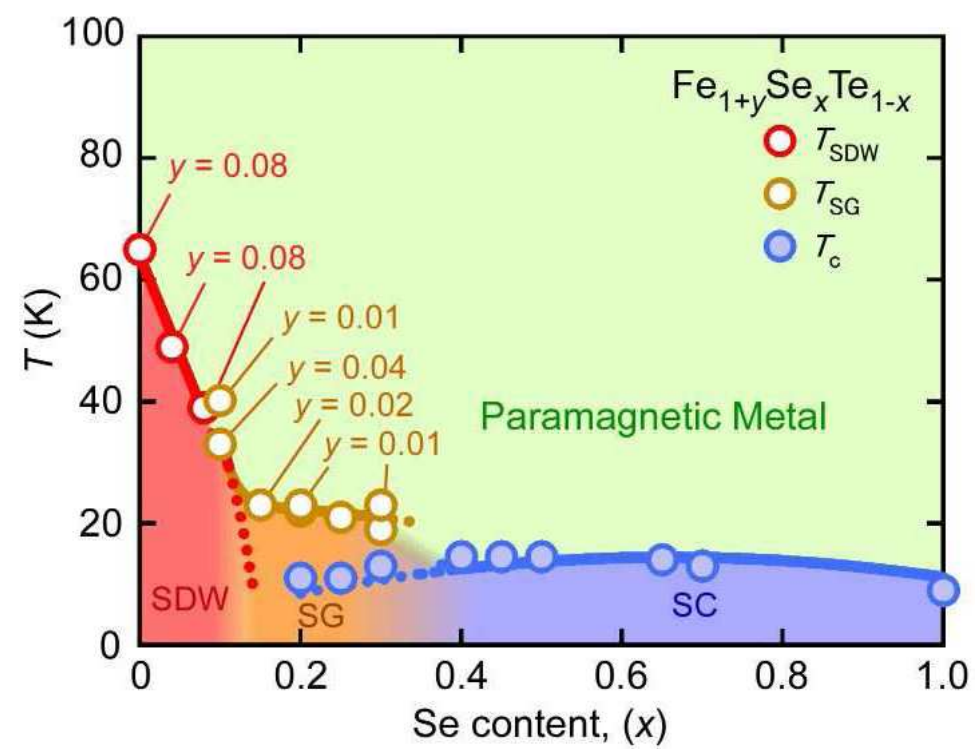

Figure 2: Phase diagram of $\mathrm{Fe}_{1+y} \mathrm{Te}_{1-x} \mathrm{Se}_{x}$, constructed from resistivity, magnetization and neutron scattering data on single crystals. The nominal Fe content is $y=0$, unless specified. SDW, SG, and SC stand for antiferromagnetic, spin-glass and superconducting phases, respectively. Reprinted from [62]. (C) 2010 Physical Society of Japan. 
It is convenient to discuss the magnetic order with reference to the phase diagram as shown in Fig. 22 (Ref. [62]). It was obtained by performing resistivity, magnetic susceptibility and neutron scattering measurements on single crystals grown using the Bridgman method 75]. Some modified versions have also been made by several other groups [59 64], but the basic features are similar to those shown in Fig. 2] At a first glance, this phase diagram is similar to those of the cuprate [5 9] and iron-pnictide superconductors [37, 46 58]. There are several important differences which need to be pointed out though: i) Unlike most other high- $T_{c}$ superconductors, where "doping" often refers to substitution of an element with another that has a different valence, here Te is isovalent to Se; ii) Superconductivity survives to $x=1$, unlike most other systems where superconductivity disappears with carrier doping above a certain value [79]; iii) The material's properties can be tuned not only by doping with Se, but also by adjusting the amount of excess Fe, so when writing the chemical formula for this system, there is a second variable. Indeed, the extra Fe is required for samples with small $x$ to stabilize the crystal structure.

$\mathrm{Fe}_{1+y} \mathrm{Te}$ is not superconducting; it exhibits coincident magnetic and structural transitions at $\sim 65 \mathrm{~K}[60,99-101]$. This behaviour is similar to that of the undoped phases of the 1111 and 122 materials [37, 46 59, 61, 63], and it is therefore often referred to as the parent compound for the 11 system [27, 99, 100, 102, 103]. Initial band-structure calculations predicted that its Fermi-surface topology should be similar to that of the iron pnictides [104]; this had been confirmed by ARPES measurements [105, 106]. From the Fermi-surface-nesting picture [104], one would expect that this system would have a collinear (C-type) spin-density-wave (SDW) order with an in-plane wave vector $(0.5,0.5)$, as shown in Fig. 3(b). However, this is in contrast to the experimental results showing a magnetic ordering vector of $(0.5,0)$ by Fruchart et al. [107] in $\mathrm{Fe}_{1.125} \mathrm{Te}$ a few decades ago. This result was confirmed by Bao et al. [99] in $\mathrm{Fe}_{1.075} \mathrm{Te}$, and by $\mathrm{Li}$ et al. [100] in $\mathrm{Fe}_{1.068} \mathrm{Te}$; each of these had a bicollinear (E-type) spin structure as shown in Fig. 3(a). Even more surprising is the fact that ARPES measurements had observed no SDW nesting instability along [0.5,0] [105, 108]. Clearly, 
a simple nesting mechanism cannot account for these experimental results.
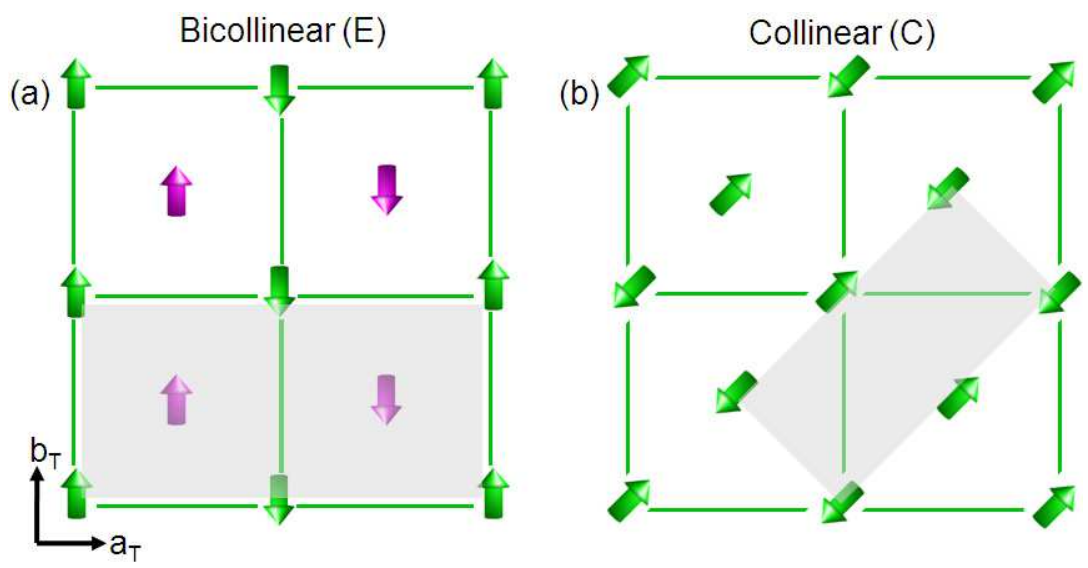

Figure 3: (a) Schematic for the bicollinear (E-type) in-plane spin structure in the tetragonal notation of the 11 compound. (b) Collinear (C-type) magnetic structure for iron pnictides. Shadow represents the magnetic unit cell.

The magnetic order in $\mathrm{Fe}_{1+y} \mathrm{Te}$ is long ranged, with a moment size of $\sim 2.5 \mu_{\mathrm{B}} / \mathrm{Fe}$ for $y=0.05$ at the base temperature [101]. The moment size is significant compared to that in the iron-pnictide antiferromagnets (less than $1 \mu_{\mathrm{B}} / \mathrm{Fe}$ [ [46, 50], but it is small compared to the effective moment estimated from the magnetic susceptibility in the paramagnetic phase [109]. The moment is found to be aligned mostly along the $b$ axis as shown in Fig. 3(a) [99 101]. The large moment together with the absence of the Fermi-surface-nesting feature along the ordering wave vector suggests that the magnetic order should be different from that of the Fe-pnictides where itinerant electrons dominate the magnetism. First-principle calculations considering the role of local moments and the importance of Hund's exchange coupling have provided better agreement with the experimental observations [49, 110 115$]$.

The order also depends on the amount of interstitial iron [99, 116 118]. The excess Fe resides in an interstitial site centered above (or below) a plaquette of four Fe sites [101, 119]. The magnetic interaction between an interstitial and the Fe nearest-neighbors results in locally ferromagnetic correlations [109, 120] and 
weak localization of the charge carriers [121]. Rodriguez et al. have constructed a magnetic-crystallographic phase diagram of $\mathrm{Fe}_{1+y} \mathrm{Te}$ [122]. They have found that the order changes as $y$ increases through the critical excess Fe content, $y_{c}$, from bicollinear commensurate to helical incommensurate [99, 100, 116, 122, 123]. The change in magnetic order correlates with the change in the lowtemperature structural symmetry from monoclinic to orthorhombic [122, 124]. A very recent theoretical calculation explains this evolution as the excess $\mathrm{Fe}$ modifying the local moments' Ruderman-Kittel-Kasuya-Yosida (RKKY) interaction, which depends on the Hund's coupling between the itinerant electrons and localized moments that is changing with $y$ [125]. It is worth mentioning that attempts utilizing spin-polarized STM to provide real-space imaging of the magnetic order in $\mathrm{Fe}_{1+y} \mathrm{Te}$ have also been made, and the results appear to be consistent with those obtained from neutron diffraction measurements [126].

Upon doping with Se, the order is suppressed, with a reduced ordering temperature (from $65 \mathrm{~K}$ to $\lesssim 30 \mathrm{~K}$ with $0.1 \mathrm{Se}$ ) [62], reduced size of the ordered magnetic moment (from $2.1 \mu_{\mathrm{B}} / \mathrm{Fe}$ to $0.27 \mu_{\mathrm{B}} / \mathrm{Fe}$ ) [60], and shorter correlation length (the magnetic peak is resolution limited in the parent compound, while with 0.25 Se doping, the order is short ranged with a correlation length of $\sim 4 \AA[99,127])$. For Se content above 0.15, there is a coexistence between the spin-glass and superconducting phase [59, 60, 62, 101, 127]. Interestingly, a magnetic peak is only observed on one side of the commensurate wave vector $(0.5,0)$, [i.e. $(0.5-\delta, 0)$ and not $(0.5 \pm \delta, 0)$ with $\delta$ being the incommensurability]; this is likely a result of an imbalance of ferromagnetic/antiferromagnetic correlations between neighbouring spins [127]. With further increase of the Se concentration, the superconductivity optimizes with $x \approx 0.5$, and no static magnetic order is observed (for $y \sim 0$ ) [59, 60, 128].

Excess Fe atoms also affect the magnetic correlations in the samples with Se doping. Xu et al. [129] have measured four samples: $\mathrm{FeTe}_{0.7} \mathrm{Se}_{0.3}, \mathrm{Fe}_{1.05} \mathrm{Te}_{0.7} \mathrm{Se}_{0.3}$; $\mathrm{FeTe}_{0.5} \mathrm{Se}_{0.5}$, and $\mathrm{Fe}_{1.05} \mathrm{Te}_{0.55} \mathrm{Se}_{0.45}$. Both samples with $y=0$ are superconducting with the same $T_{c}$ of $\sim 15 \mathrm{~K}$, although the one with $x=0.3$ has a smaller superconducting volume fraction. Also, there is short-range static magnetic or- 
der near $(0.5,0)$ in $\mathrm{FeTe}_{0.7} \mathrm{Se}_{0.3}$, while the sample with $x=0.5$ does not exhibit magnetic order, short or long ranged. With 0.05 extra Fe, superconductivity in both samples is fully suppressed, and the magnetic spectral weight around $(0.5,0)$ is strongly enhanced. In both samples, there is short-range static order. These results clearly show that the excess Fe stabilizes the antiferromagnetic order but anticorrelates with the superconductivity.

Bendele and coworkers have constructed a three-dimensional phase diagram with two variables, concentrations of Se, $x$, and excess Fe, $y$ [130]. The dependence of superconductivity and magnetic order on both $x$ and $y$ can be more clearly seen from that phase diagram [Fig. 3(b) of Ref. 130]].

Since it is very challenging to grow single crystals of $\mathrm{Fe}_{1+y} \mathrm{Te}_{1-x} \mathrm{Se}_{x}$ with $x \gtrsim 70 \%$ because of the large vapor pressure of Se, neutron scattering results on the Se-rich side have been very limited [75, 79]. In the end member $\mathrm{Fe}_{1+y} \mathrm{Se}$, although it exhibits a symmetry-lowering structural transition on cooling through $\sim 90 \mathrm{~K}$ 131 133], measurements with local probes such as Mössbauer spectroscopy [131, 134] and ${ }^{77}$ Se nuclear-magnetic-resonance (NMR) [135] indicate absence of static magnetic order. In studies of a sample that shows an increase of $T_{c}$ to $37 \mathrm{~K}$ under pressure, Mössbauer measurements indicate absence of magnetic order up to $\sim 30 \mathrm{GPa}$ [84]. In contrast, Bendele et al. [136] have reported evidence for short-range magnetic order for $P \gtrsim 0.8 \mathrm{GPa}$ based on muon-spin rotation $(\mu \mathrm{SR})$ measurements; however, this sample shows a much more modest impact of pressure on the superconductivity, with a maximum $T_{c}$ of $13 \mathrm{~K}$ at $0.7 \mathrm{GPa}$. ARPES measurements by two different groups on the thin-film samples of FeSe have detected features suggesting the existence of magnetic order for certain range of carrier dopings [88, 89]. First-principle calculations have shown that the order can be of collinear type, the same as that in the Fe-pnictides [137], or a unique block-checkerboard pattern [138]. 


\section{Spin dynamics}

\subsection{Spin dispersions}

Spin excitations in the parent compound $\mathrm{Fe}_{1+y} \mathrm{Te}$ which orders antiferromagnetically below the Néel temperature $T_{N}$ have been first reported by two groups [109, 139]. Lipscombe et al. have shown that their data can be fitted in terms of spin waves using the Heisenberg model, with anisotropic nearestneighbour $(\mathrm{NN})$ and isotropic next-nearest-neighbour (NNN) couplings [139]. In the work by Zaliznyak et al., it is found that a model with plaquettes of four ferromagnetically coaligned nearest-neighbour Fe spins that emerge as a new collective degree of freedom and have short-range antiferromagnetic correlations between the neighbouring plaquettes can more accurately describe their data [109].

There are many more reports on the spin excitations in the Se-doped materials which exhibit no long-range antiferromagnetic order. In the optimally-doped samples, the spin excitations emerge from the in-plane wave vector of $(0.5,0.5)$, different from that of the parent compound [140, 141]. The evolution of the magnetic scattering from $(0.5,0)$ to $(0.5,0.5)$ will be discussed in Sec. 3.3 The spin-excitation spectrum of a superconducting $\mathrm{FeTe}_{0.35} \mathrm{Se}_{0.65}$ sample is shown in Fig. 4(Refs. 142, 143]). The sample has a $T_{c}$ of $14 \mathrm{~K}$. In the superconducting phase [Fig. 目(a)], there is very little spectral weight below $5 \mathrm{meV}$, indicating the opening of a spin-excitation gap of $5 \pm 1 \mathrm{meV}$. The magnetic scattering appears to be commensurate for energies around $5 \mathrm{meV}$, and becomes incommensurate at higher energies, thus displaying an upward parabola shape. A number of groups have reported similar spectra [140, 141, 144, 145]. In fact, the dispersion is anisotropic, with the scattering pattern elongating only along the [1 10$]$ direction in the $(H K 0)$ plane, whereas the crystal has a four-fold symmetry [75, 141]. Lee and coworkers have shown that by taking into account the spin-orbital coupling effects, this anisotropy can be explained [141]. For high temperatures, the shape of the spectrum changes, with the scattering being clearly incommensurate at the whole energy range, and the spectrum looks like a "waterfall", as 
shown in Fig. [4(b) [142].

For samples with Se doping but without superconductivity (e.g., with more excess iron), the scattering is incommensurate, similar to that of the superconducting samples at high temperatures [146, 147]. This indicates that the incommensurate to commensurate transition of the low-energy magnetic scattering in the superconducting samples may be related to superconductivity, but intriguingly, it is found that this transition occurs at a temperature significantly higher than $T_{c}[143,147]$. By following the temperature dependence of the scattering at $5 \mathrm{meV}$ in the superconducting samples, which is centered at $(0.5,0.5)$ at low temperatures, it is shown that it splits into two incommensurate peaks displaced from $(0.5,0.5)$ at $\sim 40 \mathrm{~K}$, as shown in Fig. 4(c) [143]. Theoretically, it has been proposed that short-range ordering of the Fe $d_{x z}$ and $d_{y z}$ orbitals will promote the Fermi-surface nesting between the electron and hole pockets 148, 149]. Such a Fermi-surface topology can give rise to the commensurate scattering based on the Fermi-surface-nesting picture [104]. Under the current theoretical framework, a nested Fermi surface is necessary for superconductivity, provided that the nesting is not strong enough to induce long-range magnetic order [42, 43, 66, 150, 151]. The short-range orbital ordering disappears with increasing disorder or temperature, resulting in the incommensurate excitations as well as the suppression of superconducting correlations. Lee et al. have done detailed random phase approximation (RPA) calculations, and identified the role of orbital correlations [152]. The calculated imaginary part of the spin susceptibility agrees well with the experimental data. 152

\subsection{The resonance mode}

In this section, I will discuss the "resonance" mode in the magnetic excitations, which is probably the most prominent feature of unconventional superconductivity [153]. It describes the observation in inelastic neutron scattering experiments that the magnetic scattering at a particular $E$ and $\mathbf{Q}$ has a significant enhancement when the system enters the superconducting phase. The resonance may provide important information relevant to the pairing symme- 

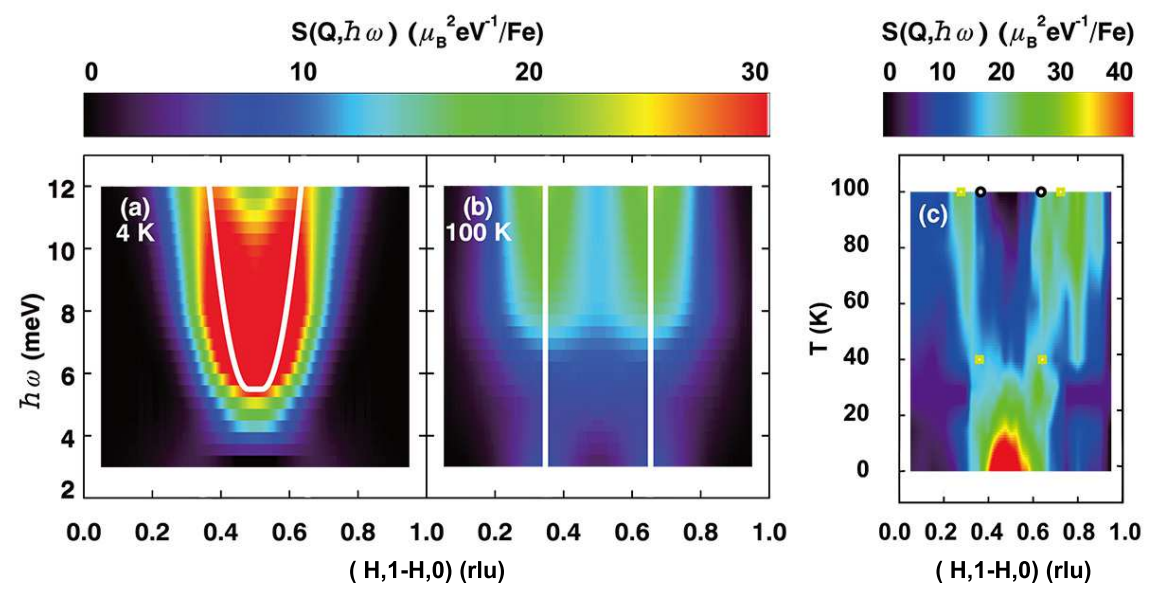

Figure 4: (a) and (b), the magnetic spectrum of a superconducting $\mathrm{FeTe}_{0.35} \mathrm{Se}_{0.65}$ sample at temperature below and well above the $T_{c}$ of $14 \mathrm{~K} ;(\mathrm{c})$, temperature dependence of the magnetic scattering at $E=\hbar \omega=5 \mathrm{meV}$. Data are extracted from Refs. [142, [143].

try, as it is predicted to occur at a particular wave vector that connects portions of the Fermi surface having opposite signs of the superconducting gap function [154 157]. Resonance excitations have been observed in a number of Fe-based superconductors [158, 159], consistent with the presumed gap-sign change between the hole and electron pockets [160]. In $\mathrm{Fe}_{1+y} \mathrm{Te}_{1-x} \mathrm{Se}_{x}$, the resonance excitation has been observed to be near the $(0.5,0.5)$ wave vector by several groups 128, 141, 144 146, 161, 162]. Note that this wave vector is rotated by $45^{\circ}$ from the magnetic ordering wave vector, whereas for iron pnictides, both magnetic order and resonance occur around the same wave vector of $(0.5,0.5)[158,159]$. In fact, it is incommensurate in $\mathbf{Q}$, peaking at $(0.5 \pm \delta, 0.5 \mp \delta)$ in a direction transverse to $(0.5,0.5)$ [141]. Compared to the magnetic excitation spectrum above $T_{c}$ [Fig. [5(b)], the low-temperature spectral weight is greatly enhanced around $(0.5,0.5)$ and the resonance energy of $\sim 6.5 \mathrm{meV}$, as shown in Fig. [5(a) 161]. The resonance is a quasi two-dimensional excitation, with little $L$ dependence [79]. The resonance energy corresponds to $\sim 5 \mathrm{k}_{\mathrm{B}} T_{c}$, similar to those of other high- $T_{c}$ superconductors [79]. Accompanying the resonance, 


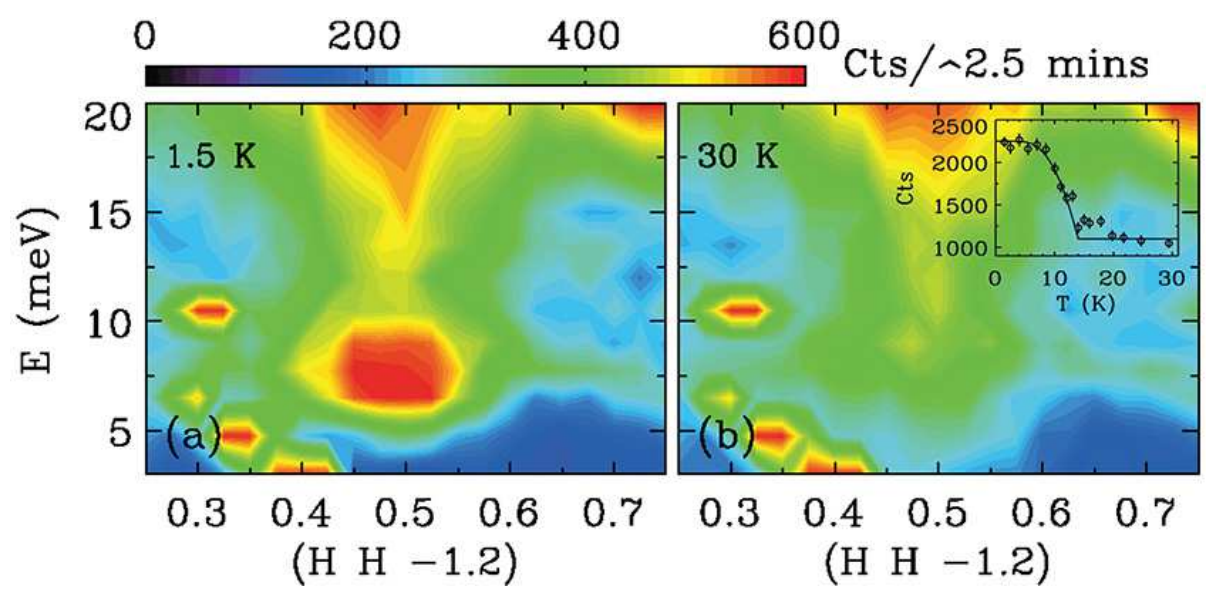

Figure 5: Spin resonance in $\mathrm{FeTe}_{0.6} \mathrm{Se}_{0.4}$. (a) and (b) show the spin excitation spectrum as a function of $\mathbf{Q}$ and energy at 1.5 and $30 \mathrm{~K}$ respectively. Inset in (b) shows the resonance intensity as a function of temperature. Data are obtained from Ref. [161].

there is a spin gap with an energy of $5 \pm 1 \mathrm{meV}$; the intensity below the spin gap is shifted to the resonance in the superconducting state [128, 161]. The temperature dependence of the resonance intensity looks like the superconducting order parameter, as shown in the inset of Fig. 5(b), demonstrating its close relationship with superconductivity.

Since superconductivity can be suppressed by an external magnetic field, one will naturally expect that the field should impact the resonance if it is related to the pairing, as seen in $\mathrm{YBa}_{2} \mathrm{Cu}_{3} \mathrm{O}_{6.6}$ [163] and in $\mathrm{La}_{1.82} \mathrm{Sr}_{0.18} \mathrm{CuO}_{4}$ [164]. Experiment on $\mathrm{FeTe}_{0.5} \mathrm{Se}_{0.5}$ indeed gives positive answers [128]. It is shown that under an external field of $7 \mathrm{~T}$, the resonance starts to appear at a lowered $T_{c}$, $12 \mathrm{~K}$, with reduced intensity, coincident with the suppression of the superconductivity; however, there is no detectable change in either the resonance energy or the width of the resonance peak [128]. With a field of $14.5 \mathrm{~T}$, Zhao et al. [165] have shown that in $\mathrm{BaFe}_{1.9} \mathrm{Ni}_{0.1} \mathrm{As}_{2}$, both the resonance energy and intensity are reduced, and the resonance peak is slightly broadened.

It is believed by many researchers that the spin resonance is a singlet-totriplet excitation of the Cooper pairs [156, 157, 166]. In principle, inelastic 
neutron scattering experiments under magnetic field should be able to test this hypothesis. A magnetic field should induce a Zeeman splitting and lift the degeneracy of the triplet excited state [163]. Bao et al. 167] have tried to address this problem by applying a 14-T field on $\mathrm{FeSe}_{0.4} \mathrm{Te}_{0.6}$, and it appears that the field induces two additional peaks, consistent with the assumption that the resonance is a triplet. However, a more recent experiment shows that the field only reduces the spectral weight around the resonance mode [168]. Perhaps relevant to this debate is a recent magnetic-field study on the resonance in the heavy-fermion superconductor $\mathrm{CeCoIn}_{5}$, which reports that the resonance is a doublet, as the field splits it into two peaks [169]. This result is however in contrast to the earlier studies where only reduction of the resonance energy is found $[170,171]$. With no doubts, the debates on the nature of the resonance mode will be continued.

\subsection{Doping evolution of the magnetic excitations}

As shown in Sec. 2, the non-superconducting parent compound $\mathrm{Fe}_{1+y} \mathrm{Te}$ has static magnetic order with a bicollinear spin configuration. In these samples, spin excitations emerge from $(0.5,0)$. In samples with robust superconducting properties, there is strong magnetic scattering around $(0.5,0.5)$ with a spin resonance below $T_{c}$. The excitations correspond to spin correlations of the collinear type. It is natural to ask how the spin dynamics evolve from the parent compound to the optimally-doped materials. A number of theoretical [113, 115] and experimental [60, 129, 140, 146] studies have been carried out on the doping evolution of the magnetic correlations (static and dynamic) and their connection with superconductivity. Lumsden et al. [140] have performed measurements using TOFS on two samples, a non-superconducting $\mathrm{Fe}_{1.04} \mathrm{Te}_{0.73} \mathrm{Se}_{0.27}$ sample and a superconducting $\mathrm{FeTe}_{0.51} \mathrm{Se}_{0.49}$ sample. It is clearly shown in Fig. 6(a) that at 5-7 meV, for the non-superconducting sample, the spectral weight is mostly concentrated around $(0.5,0)$, where static magnetic order is observed. For the superconducting sample, magnetic excitations near $(0.5,0.5)$ are dominant, as shown in Fig. 6(b). On the other hand, the high-energy (larger than 
$120 \mathrm{meV}$ ) spectrum looks qualitatively similar for these two samples [140]. Several other groups have shown similar results that support the same conclusions: the spectral weight around $(0.5,0)$ is related to the static magnetic order that competes with superconductivity, while the collinear spin correlations appear to be strengthened as superconductivity is optimized [60, 129, 146, 147].
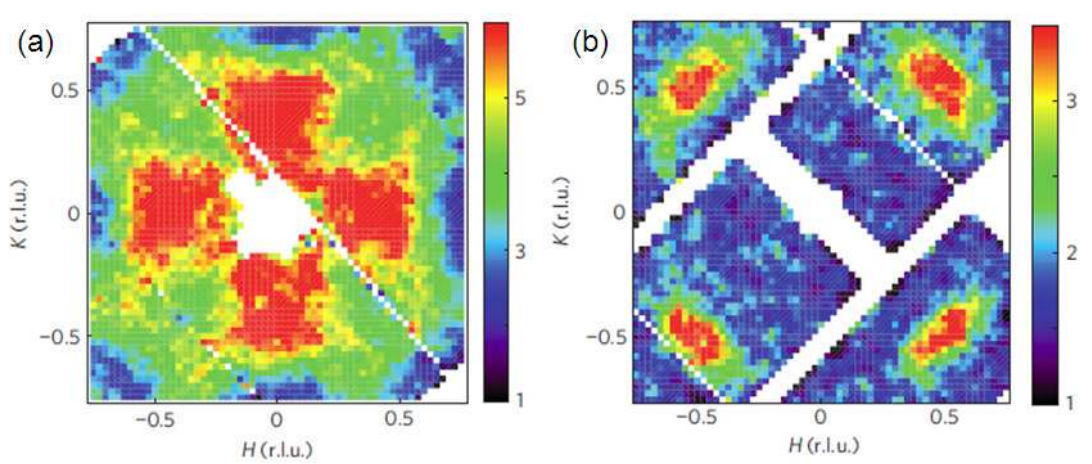

Figure 6: (a) Constant-energy cut of the magnetic excitation spectrum at an energy transfer of $6 \pm 1 \mathrm{meV}$ for the non-superconducting sample $\mathrm{Fe}_{1.04} \mathrm{Te}_{0.73} \mathrm{Se}_{0.27}$ at $5 \mathrm{~K}$. (b) Plot for the superconducting sample $\mathrm{FeTe}_{0.51} \mathrm{Se}_{0.49}$ at $3.5 \mathrm{~K}$. Reprinted with permission from [140]. (c) 2010 Macmillan Publishers Ltd.

The second variable $y$ in $\mathrm{Fe}_{1+y} \mathrm{Te}_{1-x} \mathrm{Se}_{x}$ also affects the magnetic excitations. By systematically studying the effects of excess Fe, it is shown in Ref. [129] that increasing excess Fe acts like reducing the amount of Se in terms of tuning the spin excitations - with more Fe, the low-energy excitations close to $(0.5,0)$ are enhanced, while those close to $(0.5,0.5)$ are suppressed, along with superconductivity. The extra Fe can induce short-range static magnetic order in the samples where no such order is present. These results reinforce the idea that the bicollinear E-type spin correlations are harmful for superconductivity, while the collinear C-type correlations may be compatible with it. In the parent compound $\mathrm{Fe}_{1+y} \mathrm{Te}$, Stock et al. 172] have also shown that by changing $y$, the low-energy magnetic excitation spectrum can be dramatically modified, thus demonstrating the important role of excess Fe. 


\section{Nature of the magnetism}

As in the cuprates, the origin of the magnetism in the Fe-based compounds is heatedly debated [173]. There are three main scenarios on this debate. First, there is the localized-moment picture, where strong correlation and Hund's coupling dominate 112, 174, 175]. Second, there is the opposite case, where itinerant electrons contribute to the moments at low temperature as a result of the SDW instability, which depends critically on the nesting properties of the Fermi surface [42, 66, 104, 110, 121, 176-180]. Given the metallic behavior of the Fe-pnictides, this idea is generally more favorable. A third possibility is intermediate between these two cases, where some of the $d$-electrons are indeed itinerant, while others are localized, giving rise to an intermediate-size moment 76, 125, 181, 182]. In addition to these three cases, there is the possibility that none of these is correct, but instead, that the magnetism derives simply from the energy gain associated with transfer to lower energy of one-electron density-of-states (DOS) spectral weight [49].

For $\mathrm{Fe}_{1+y} \mathrm{Te}_{1-x} \mathrm{Se}_{x}$, opinions are more divided compared with other Fe-based superconductors [108, 183], as it has stronger electronic correlation [184], larger magnetic moment [99], and poorer metallic properties [75]. More importantly, as discussed in Sec. 2, a simple nesting picture in an itinerant electron model cannot describe the magnetic order [105]. However, for the magnetic excitations around $(0.5,0.5)$, Argyriou et al. have shown that the incommensurate spin excitation spectrum can be fitted well assuming that the excitations are quasiparticle scatterings between the hole and electron bands [145], but the lack of temperature dependence of the spectral weight and the large moment size do not agree with the itinerant model [142]. X-ray emission spectroscopy measurements on $\mathrm{Fe}_{1+y} \mathrm{Te}_{1-x} \mathrm{Se}_{x}$ suggest that the magnetism has a dual nature, with contributions from both localized spins and itinerant electrons [185]. This is consistent with the transport and magnetic susceptibility results that show both itinerant electrons and local moments exist in the system [26, 186]. These two components are further shown not to be isolated, but instead entangled [109]. 


\section{Substitution effects of $3 d$ transition metals}

There have been extensive studies on the effects of substitution with $3 d$ transition metals (such as $\mathrm{Co}, \mathrm{Ni}, \mathrm{Cu}$, etc) in the Fe-based superconductors $187-$ 190]. Some initial studies on $\mathrm{BaFe}_{2} \mathrm{As}_{2}$ (Ba122) have suggested that $3 d$ metals such as $\mathrm{Co}$, when partially substituted for $\mathrm{Fe}$, act as effective electron donors 187, 190, 191]; this process can be described using a rigid-band shift model $192-194]$. However, such a model has faced serious challenges from both experimental and theoretical perspectives [188, 189, 195 197]. Furthermore, there is a dichotomy between $\mathrm{Co} / \mathrm{Ni}$ and $\mathrm{Cu}$ substitution effects [187, 188, 190, 191, 196, 198, 199]. In $\mathrm{Fe}_{1+y} \mathrm{Te}_{1-x} \mathrm{Se}_{x}, \mathrm{Cu}$ is also found to have a stronger suppression on both the conductivity and superconductivity than Co and Ni 80, 200 206].

The author and collaborators have performed resistivity and inelastic neutron scattering measurements on three samples of $\mathrm{Fe}_{0.98-z} \mathrm{Cu}_{z} \mathrm{Te}_{0.5} \mathrm{Se}_{0.5}$ with $z=0,0.02$, and 0.1 (labelled $\mathrm{Cu} 0, \mathrm{Cu} 02$, and $\mathrm{Cu} 10$ respectively) [207]. It is found that with increasing $\mathrm{Cu}$ doping the sample's resistivity deviates progressively from that of a metal. The $\mathrm{Cu} 02$ (in the normal state) and Cu10 samples behave like three-dimensional Mott insulators, similar to the behavior in $\mathrm{Cu}$ doped FeSe for $\mathrm{Cu}$ doping larger than $4 \%$ [201, 202]. Meanwhile, the low-energy $(\leq 12 \mathrm{meV})$ magnetic scattering is enhanced in strength, with greater spectral weight and longer dynamical spin-spin correlation lengths. Such an enhancement of the low-energy magnetic scatterings by $\mathrm{Cu}$ doping is intriguing. One plausible interpretation of the results is that the main effect of $\mathrm{Cu}$ substitution is to introduce localization into the system and suppress the itinerancy, and thus to enhance the magnetic correlations [175, 199]. In the case of $\mathrm{Cu}-$ doped FeSe, it has also been suggested that $\mathrm{Cu}$ substitution introduces local moments, and when $\mathrm{Cu}$ doping equals 0.12 , the sample exhibits a spin-glass transition [201]. More recently, our group have also found evidence that $\mathrm{Co} / \mathrm{Ni}$ and $\mathrm{Cu}$ have different effects on magnetic excitations [208], consistent with the different responses of transport properties to these impurities [200 206]. 
The effects of $\mathrm{Cu}$ substitution in the parent compound $\mathrm{Fe}_{1.1}$ Te have also been studied [209]. It is found that $\mathrm{Cu}$ drives down the structural and magnetic transitions, with long-range nearly-commensurate magnetic order retained in $\mathrm{Fe}_{1.06} \mathrm{Cu}_{0.04} \mathrm{Te}$, but only short-range incommensurate order in $\mathrm{FeCu}_{0.1} \mathrm{Te}[209]$. In the latter sample, the structural phase transition is not obvious and a transition to a spin-glass state is found at $22 \mathrm{~K}[209]$. In $\mathrm{Fe}_{1.06} \mathrm{Cu}_{0.04} \mathrm{Te}$, the initial structural and magnetic ordering occurs at $41 \mathrm{~K}$, involving short-range incommensurate order that abruptly shifts to long-range nearly-commensurate order below $36 \mathrm{~K}$ [209]. Inelastic scattering measurements indicate a spin anisotropy gap of $4.5 \mathrm{meV}$ in the nearly-commensurate phase [209]. The results are consistent with the idea that the frustration of the exchange interactions between the coupled Fe spins increases as more $\mathrm{Cu}$ is added. Although both the structural phase transition and magnetic order are suppressed with increasing $\mathrm{Cu}$ substitution, superconductivity is not observed [209]. The key issue is likely associated with the spin configurations. Although there is a suppression of the magnetic order at $(0.5,0)$, no evidence of the enhancement of the C-type spin correlations has been observed. This is another piece of evidence for the importance of low-energy fluctuations near $(0.5,0.5)$ to the superconductivity in $\mathrm{Fe}_{1+y} \mathrm{Te}_{1-x} \mathrm{Se}_{x}$.

\section{Summary}

To summarize, I review the progress of neutron scattering studies on the Fe-based superconductor $\mathrm{Fe}_{1+y} \mathrm{Te}_{1-x} \mathrm{Se}_{x}$. Many important results concerning the relationship between spin and superconductivity have been reported thus far. Particularly, while the bicollinear spin correlations appear to compete with superconductivity, the collinear correlations seem to promote it. I have also shown that both local moments and itinerant electrons may contribute to magnetic excitations. Substitution with different $3 d$ transition metals may have varying effects in the 11 system; for example, the main effect of $\mathrm{Cu}$ is to induce localization. 
Although the research pace has been extremely fast, there still remain many challenges. For example, it appears relatively easy to make large single crystals for $\mathrm{Fe}_{1+y} \mathrm{Te}_{1-x} \mathrm{Se}_{x}(x \leq 0.7)$, but it is difficult to have them be homogeneous 210 212]. Also, the extra Fe can sometimes complicate interpretation. Therefore, homogeneous samples with more precisely controlled stoichiometry may provide a better understanding of the physics. This goal may be achieved by using a smaller cooling rate during the growth, or annealing the as-grown crystals $\underline{64}$, 213 216]. Given the many interesting reports on the thin-film samples of FeSe [87 92], it is certainly worth putting more effort to growing single crystals of FeSe. Recent studies have reported the growth of phase pure crystals of superconducting FeSe [217, 218]. On the measurement side, there are still many things that can be done. For example, the origin of the resonance and the magnetism are still not fully understood, and require further investigation.

\section{Acknowledgements}

The work at Nanjing University was supported by National Natural Science Foundation of China under Contract No. 11374143, Ministry of Education under Contract No. NCET-13-0282, and Fundamental Research Funds for the Central Universities. I thank all of the collaborators listed in the references, especially those at Brookhaven National Laboratory and University of California at Berkeley, where I had worked; and the colleagues in the Physics Department of Nanjing University, where I am now affiliated. I am grateful for the colleagues and collaborators for allowing their work to be reproduced here. I thank J. A. Schneeloch for proof reading.

\section{References}

\section{References}

[1] J. G. Bednorz, K. A. Müller, Possible high $t_{c}$ superconductivity in the ba-la-cu-o system, Z. Phys. B 64 (1986) 189. 
[2] M. K. Wu, J. R. Ashburn, C. J. Torng, P. H. Hor, R. L. Meng, L. Gao, Z. J. Huang, Y. Q. Wang, C. W. Chu, Superconductivity at $93 \mathrm{k}$ in a new mixed-phase y-ba-cu-o compound system at ambient pressure, Phys. Rev. Lett. 58 (1987) 908.

[3] Z. X. Zhao, L. Q. Chen, Q. S. Yang, Y. Z. Huaug, G. H. Chen, R. M. Tang, G. R. Liu, C. G. Cui, L. Chen, L. H. Wang, S. Q. Guo, S. L. Li, J. Q. $\mathrm{Bi}$, Superconductivity at $93 \mathrm{k}$ in a new mixed-phase y-ba-cu-o compound system at ambient pressure, Kexue Tongbao 6 (1987) 412.

[4] J. Bardeen, L. N. Cooper, J. R. Schrieffer, Theory of superconductivity, Phys. Rev. 108 (1957) 1175.

[5] P. A. Lee, N. Nagaosa, X.-G. Wen, Doping a mott insulator: Physics of high-temperature superconductivity, Rev. Mod. Phys. 78 (2006) 17.

[6] E. W. Carlson, V. J. Emery, S. A. Kivelson, D. Orgad, The Physics of Conventional and Unconventional Superconductors, Springer-Verlag, 2002, Ch. Concepts in High Temperature Superconductivity.

[7] R. J. Birgeneau, C. Stock, J. M. Tranquada, K. Yamada, Magnetic neutron scattering in hole doped cuprate superconductors, J. Phys. Soc. Jpn. 75 (2006) 111003.

[8] M. A. Kastner, R. J. Birgeneau, G. Shirane, Y. Endoh, Magnetic, transport, and optical properties of monolayer copper oxides, Rev. Mod. Phys. 70 (1998) 897.

[9] J. Orenstein, A. J. Millis, Advances in the physics of high-temperature superconductivity, Science 288 (2000) 468.

[10] Y. Kamihara, H. Hiramatsu, M. Hirano, R. Kawamura, H. Yanagi, T. Kamiya, H. Hosono, Iron-based layered superconductor: Laofep, J. Am. Chem. Soc. 128 (2006) 10012. 
[11] Y. Kamihara, T. Watanabe, M. Hirano, H. Hosono, Iron-based layered superconductor la $\left[\mathrm{O}_{1-x} \mathrm{f}_{x}\right]$ feas $(\mathrm{x}=0.05-0.12)$ with $t_{c}=26 \mathrm{k}, \mathrm{J}$. Am. Chem. Soc. 130 (2008) 3296.

[12] A. S. Sefat, R. Jin, M. A. McGuire, B. C. Sales, D. J. Singh, D. Mandrus, Superconductivity at $22 \mathrm{k}$ in co-doped bafe $\mathrm{as}_{2}$ crystals, Phys. Rev. Lett. 101 (2008) 117004.

[13] M. Rotter, M. Tegel, D. Johrendt, Superconductivity at $38 \mathrm{k}$ in the iron arsenide ba ${ }_{1-x} \mathrm{k}_{x} \mathrm{fe}_{2} \mathrm{as}_{2}$, Phys. Rev. Lett. 101 (2008) 107006.

[14] G. F. Chen, Z. Li, G. Li, W. Z. Hu, J. Dong, X. D. Zhang, P. Zheng, N. L. Wang, J. L. Luo, Superconductivity in hole-doped $\left(\mathrm{sr}_{1-x} \mathrm{k}_{x}\right) \mathrm{fe}_{2} \mathrm{as}_{2}$, Chin. Phys. Lett. 25 (2008) 3403.

[15] D. S. Inosov, A. Leineweber, X. Yang, J. T. Park, N. B. Christensen, R. Dinnebier, G. L. Sun, C. Niedermayer, D. Haug, P. W. Stephens, J. Stahn, O. Khvostikova, C. T. Lin, O. K. Andersen, B. Keimer, V. Hinkov, Suppression of the structural phase transition and lattice softening in slightly underdoped $\mathrm{ba}_{1-x} \mathrm{k}_{x} \mathrm{fe}_{2} \mathrm{as}_{2}$ with electronic phase separation, Phys. Rev. B 79 (2009) 224503.

[16] Z. Deng, X. C. Wang, Q. Liu, S. J. Zhang, Y. X. Lv, J. L. Zhu, R. Yu, C. Jin, A new 111 type iron pnictide superconductor lifep, Euro. Phys. Lett. 87 (2009) 3704.

[17] X. C. Wang, Q. Q. Liu, Y. X. Lv, W. B. Gao, L. X. Yang, R. C. Yu, F. Y. Li, C. Q. Jin, The superconductivity at $18 \mathrm{~K}$ in LiFeAs system, Solid State Commun. 148 (2008) 538.

[18] M. J. Pitcher, D. R. Parker, P. Adamson, S. J. C. Herkelrath, A. T. Boothroyd, S. J. Clarke, Structure and superconductivity of LiFeAs, Chem. Commun. 45 (2008) 5918. 
[19] C. W. Chu, F. Chen, M. Gooch, A. M. Guloy, B. Lorenz, B. Lv, K. Sasmal, Z. J. Tang, J. H. Tapp, Y. Y. Xue, The synthesis and characterization of LiFeAs and NaFeAs, Physica C 469 (2009) 326.

[20] J. H. Tapp, Z. Tang, B. Lv, K. Sasmal, B. Lorenz, P. C. W. Chu, A. M. Guloy, Lifeas: An intrinsic feas-based superconductor with $t_{c}=18 \mathrm{k}$, Phys. Rev. B 78 (2008) 060505.

[21] Z. Deng, X. C. Wang, Q. Q. Liu, S. J. Zhang, Y. X. Lv, J. L. Zhu, R. C. Yu, C. Q. Jin, A new "111" type iron pnictide superconductor LiFeP, Euro. Phys. Lett. 87 (2009) 37004.

[22] S. J. Zhang, X. C. Wang, Q. Q. Liu, Y. X. Lv, X. H. Yu, Z. J. Lin, Y. S. Zhao, L. Wang, Y. Ding, H. K. Mao, C. Q. Jin, Superconductivity at 31 $\mathrm{K}$ in the "111"-type iron arsenide superconductor $\mathrm{Na}_{1-x} \mathrm{FeAs}$ induced by pressure, Euro. Phys. Lett. 88 (2009) 47008.

[23] F.-C. Hsu, J.-Y. Luo, K.-W. Yeh, T.-K. Chen, T.-W. Huang, P. M. Wu, Y.-C. Lee, Y.-L. Huang, Y.-Y. Chu, D.-C. Yan, M.-K. Wu, Superconductivity in the pbo-type structure alpha-fese, Proc. Natl. Acad. Sci. USA 105 (2008) 14262.

[24] K.-W. Yeh, T.-W. Huang, Y.-L. Huang, T.-K. Chen, F.-C. Hsu, P. M. Wu, Y.-C. Lee, Y.-Y. Chu, C.-L. Chen, J.-Y. Luo, D.-C. Yan, M. kuen $\mathrm{Wu}$, Tellurium substitution effect on superconductivity of the $\alpha$-phase iron selenide, Euro. Phys. Lett. 84 (2008) 37002.

[25] B. C. Sales, A. S. Sefat, M. A. McGuire, R. Y. Jin, D. Mandrus, Y. Mozharivskyj, Bulk superconductivity at $14 \mathrm{k}$ in single crystals of $\mathrm{fe}_{1+y} \mathrm{te}_{x} \mathrm{se}_{1-x}$, Phys. Rev. B 79 (2009) 094521.

[26] G. F. Chen, Z. G. Chen, J. Dong, W. Z. Hu, G. Li, X. D. Zhang, P. Zheng, J. L. Luo, N. L. Wang, Electronic properties of single-crystalline fe $e_{1.05}$ te and $\mathrm{fe}_{1.03} \mathrm{se}_{0.30} \mathrm{te}_{0.70}$, Phys. Rev. B 79 (2009) 140509(R). 
[27] M. H. Fang, H. M. Pham, B. Qian, T. J. Liu, E. K. Vehstedt, Y. Liu, L. Spinu, Z. Q. Mao, Superconductivity close to magnetic instability in fe $\left(\mathrm{se}_{1-x} \mathrm{te}_{x}\right)_{0.82}$, Phys. Rev. B 78 (2008) 224503.

[28] X. Zhu, F. Han, G. Mu, P. Cheng, B. Shen, B. Zeng, H.-H. Wen, Transition of stoichiometric $\mathrm{sr}_{2} \mathrm{VO}_{3}$ feas to a superconducting state at $37.2 \mathrm{k}$, Phys. Rev. B 79 (2009) 220512.

[29] H. Ogino, Y. Matsumura, Y. Katsura, K. Ushiyama, S. Horii, K. Kishio, J. ichi Shimoyama, Superconductivity at $17 \mathrm{k}$ in $\left(\mathrm{fe}_{2} \mathrm{p}_{2}\right)\left(\mathrm{sr}_{4} \mathrm{Sc}_{2} \mathrm{O}_{6}\right)$ : a new superconducting layered pnictide oxide with a thick perovskite oxide layer, Supercon. Sci. Tech. 22 (2009) 075008.

[30] J. Guo, S. Jin, G. Wang, S. Wang, K. Zhu, T. Zhou, M. He, X. Chen, Superconductivity in the iron selenide $\mathrm{k}_{x} \mathrm{fe}_{2} \mathrm{Se}_{2}(0 \leq x \leq 1.0)$, Phys. Rev. B 82 (2010) 180520.

[31] Y. Mizuguchi, H. Takeya, Y. Kawasaki, T. Ozaki, S. Tsuda, T. Yamaguchi, Y. Takano, Transport properties of the new Fe-based superconductor $\mathrm{K}_{x} \mathrm{Fe}_{2} \mathrm{Se}_{2}\left(T_{c}=33 \mathrm{~K}\right)$, Appl. Phys. Lett. 98 (2011) 042511.

[32] A. F. Wang, J. J. Ying, Y. J. Yan, R. H. Liu, X. G. Luo, Z. Y. Li, X. F. Wang, M. Zhang, G. J. Ye, P. Cheng, Z. J. Xiang, X. H. Chen, Superconductivity at $32 \mathrm{k}$ in single-crystalline $\mathrm{rb}_{x} \mathrm{fe}_{2-y} \mathrm{se}_{2}$, Phys. Rev. B 83 (2011) 060512.

[33] A. Krzton-Maziopa, Z. Shermadini, E. Pomjakushina, V. Pomjakushin, M. Bendele, A. Amato, R. Khasanov, H. Luetkens, K. Conder, Synthesis and crystal growth of $\mathrm{cs}_{0.8}\left(\text { fese }_{0.98}\right)_{2}$ : a new iron-based superconductor with $t_{c}=27 \mathrm{k}$, J. Phys. Conden. Matter 23 (2011) 052203.

[34] H.-D. Wang, C.-H. Dong, Z.-J. Li, Q.-H. Mao, S.-S. Zhu, C.-M. Feng, H. Q. Yuan, M.-H. Fang, Superconductivity at $32 \mathrm{k}$ and anisotropy in $\mathrm{tl}_{0.58} \mathrm{rb}_{0.42} \mathrm{fe}_{1.72} \mathrm{se}_{2}$ crystals, Euro. Phys. Lett. 93 (2011) 47004. 
[35] J. Zhao, Q. Huang, C. de la Cruz, J. W. Lynn, M. D. Lumsden, Z. A. Ren, J. Yang, X. Shen, X. Dong, Z. Zhao, P. Dai, Lattice and magnetic structures of prfeaso, prfeaso $0_{0.85} \mathrm{f}_{0.15}$, and prfeaso $_{0.85}$, Phys. Rev. B 78 (2008) 132504.

[36] C. Lester, J.-H. Chu, J. G. Analytis, S. C. Capelli, A. S. Erickson, C. L. Condron, M. F. Toney, I. R. Fisher, S. M. Hayden, Neutron scattering study of the interplay between structure and magnetism in ba(fe $\left.{ }_{1-x} \mathrm{co}_{x}\right)_{2}$ as $_{2}$, Phys. Rev. B 79 (2009) 144523.

[37] Y. Qiu, W. Bao, Q. Huang, T. Yildirim, J. M. Simmons, M. A. Green, J. W. Lynn, Y. C. Gasparovic, J. Li, T. Wu, G. Wu, X. H. Chen, Crystal structure and antiferromagnetic order in ndfeaso ${ }_{1-x} \mathrm{f}_{x}(\mathrm{x}=0.0$ and 0.2 ) superconducting compounds from neutron diffraction measurements, Phys. Rev. Lett. 101 (2008) 257002.

[38] N. Kumar, S. Chi, Y. Chen, K. G. Rana, A. K. Nigam, A. Thamizhavel, W. Ratcliff, II, S. K. Dhar, J. W. Lynn, Evolution of the bulk properties, structure, magnetic order, and superconductivity with ni doping in cafe $_{2-x} \mathrm{ni}_{x}$ as $_{2}$, Phys. Rev. B 80 (2009) 144524.

[39] C. Wang, L. Li, S. Chi, Z. Zhu, Z. Ren, Y. Li, Y. Wang, X. Lin, Y. Luo, S. Jiang, X. Xu, G. Cao, Z. Xu, Thorium-doping-induced superconduc-

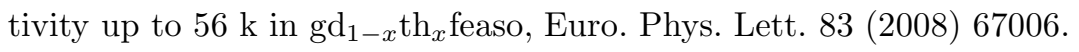

[40] Z.-A. Ren, W. Lu, J. Yang, W. Yi, X.-L. Shen, Z.-C. Li, G.-C. Che, X.-L. Dong, L.-L. Sun, F. Zhou, Z.-X. Zhao, Superconductivity at 55 k in ironbased f-doped layered quaternary compound $\mathrm{smo}_{1-x} \mathrm{f}_{x}$ feas, Chin. Phys. Lett. 25 (2008) 2215.

[41] F. Ma, Z.-Y. Lu, Iron-based layered compound lafeaso is an antiferromagnetic semimetal, Phys. Rev. B 78 (2008) 033111.

[42] I. I. Mazin, D. J. Singh, M. D. Johannes, M. H. Du, Unconventional super- 
conductivity with a sign reversal in the order parameter of lafeaso ${ }_{1-x} \mathrm{f}_{x}$, Phys. Rev. Lett. 101 (2008) 057003.

[43] K. Kuroki, S. Onari, R. Arita, H. Usui, Y. Tanaka, H. Kontani, H. Aoki, Unconventional pairing originating from the disconnected fermi surfaces of superconducting lafeaso ${ }_{1-x} \mathrm{f}_{x}$, Phys. Rev. Lett. 101 (2008) 087004.

[44] H. Kontani, S. Onari, Orbital-fluctuation-mediated superconductivity in iron pnictides: Analysis of the five-orbital hubbardholstein model, Phys. Rev. Lett. $104 \quad$ (15) (2010) 157001. doi:10.1103/PhysRevLett.104.157001

[45] I. Mazin, Superconductivity gets an iron boost, Nature 464 (2010) 183.

[46] C. de la Cruz, Q. Huang, J. W. Lynn, J. Li, W. Ratcliff, J. L. Zarestzky, H. A. Mook, G. F. Chen, J. L. Luo, N. L. Wang, P. Dai, Magnetic order close to superconductivity in the iron-based layered $\operatorname{lao}_{1-x} \mathrm{f}_{x}$ feas systems, Nature 453 (2008) 899.

[47] Q. Huang, Y. Qiu, W. Bao, M. A. Green, J. W. Lynn, Y. C. Gasparovic, T. Wu, G. Wu, X. H. Chen, Neutron-diffraction measurements of magnetic order and a structural transition in the parent bafe as $_{2}$ compound of feas-based high-temperature superconductors, Phys. Rev. Lett. 101 (2008) 257003.

[48] Y. Chen, J. W. Lynn, J. Li, G. Li, G. F. Chen, J. L. Luo, N. L. Wang, P. Dai, C. de la Cruz, H. A. Mook, Magnetic order of the iron spins in ndfeaso, Phys. Rev. B 78 (2008) 064515.

[49] M. D. Johannes, I. I. Mazin, Microscopic origin of magnetism and magnetic interactions in ferropnictides, Phys. Rev. B 79 (2009) 220510(R).

[50] S. D. Wilson, Z. Yamani, C. R. Rotundu, B. Freelon, E. BourretCourchesne, R. J. Birgeneau, Neutron diffraction study of the magnetic and structural phase transitions in bafe $a_{2} a_{2}$, Phys. Rev. B 79 (2009) 184519. 
[51] M. Kofu, Y. Qiu, W. bao, S. H. Lee, S. Chang, T. Wu, G. Wu, X. H. Chen, Neutron scattering investigation of the magnetic order in single crystalline bafe $_{2}$ as $_{2}$, New J. Phys. 11 (2009) 055001.

[52] J. Zhao, Q. Huang, C. de la Cruz, S. Li, J. W. Lynn, Y. Chen, M. A. Green, G. F. Chen, G. Li, Z. Li, J. L. Luo, N. L. Wang, , P. Dai, Structural and magnetic phase diagram of cefeaso ${ }_{1-x} f_{x}$ and its relation to hightemperature superconductivity, Nature Mater. 7 (2008) 953.

[53] H. Luetkens, H. H. Klauss, M. Kraken, F. J. Litterst, T. Dellmann, R. Klingeler, C. Hess, R. Khasanov, A. Amato, C. Baines, J. HamannBorrero, N. Leps, A. Kondrat, G. Behr, J. Werner, B. Buechner, The electronic phase diagram of the $\mathrm{lao}_{1-x} \mathrm{f}_{x}$ feas superconductor, Nature Mater. 8 (2009) 305.

[54] A. J. Drew, C. Niedermayer, P. J. Baker, F. L. Pratt, S. J. Blundell, T. Lancaster, R. H. Liu, G. Wu, X. H. Chen, I. Watanabe, V. K. Malik, A. Dubroka, M. Roessle, K. W. Kim, C. Baines, C. Bernhard, Coexistence of static magnetism and superconductivity in $\operatorname{smfeaso}_{1-x} \mathrm{f}_{x}$ as revealed by muon spin rotation, Nature Mater. 8 (2009) 310.

[55] M. Rotter, M. Pangerl, M. Tegel, D. Johrendt, Superconductivity and crystal structures of $\left(\mathrm{ba}_{1-x} \mathrm{k}_{x}\right) \mathrm{fe}_{2} \mathrm{as}_{2}(\mathrm{x}=0$ - 1), Angew. Chem. Int.. Ed. 47 (2008) 7949.

[56] H. Chen, Y. Ren, Y. Qiu, W. bao, R. H. Liu, G. Wu, T. Wu, Y. L. Xie, X. F. Wang, Q. Huang, X. H. Chen, Coexistence of the spin-density-wave and superconductivity in the (ba,k)fe $\mathrm{fes}_{2}$, Euro. Phys. Lett. 85 (2009) 17006 .

[57] L. Fang, H. Luo, P. Cheng, Z. Wang, Y. Jia, G. Mu, B. Shen, I. I. Mazin, L. Shan, C. Ren, H.-H. Wen, Roles of multiband effects and electronhole asymmetry in the superconductivity and normal-state properties of ba(fe $\left.{ }_{1-x} \mathrm{CO}_{x}\right)_{2} \mathrm{as}_{2}$, Phys. Rev. B 80 (2009) 140508(R). 
[58] J.-H. Chu, J. G. Analytis, C. Kucharczyk, I. R. Fisher, Determination of the phase diagram of the electron-doped superconductor ba $\left(\mathrm{fe}_{1-x} \mathrm{CO}_{x}\right)_{2} \mathrm{as}_{2}$, Phys. Rev. B 79 (2009) 014506.

[59] R. Khasanov, M. Bendele, A. Amato, P. Babkevich, A. T. Boothroyd, A. Cervellino, K. Conder, S. N. Gvasaliya, H. Keller, H.-H. Klauss, H. Luetkens, V. Pomjakushin, E. Pomjakushina, B. Roessli, Coexistence of incommensurate magnetism and superconductivity in $\mathrm{fe}_{1+y} \mathrm{se}_{x} \mathrm{te}_{1-x}$, Phys. Rev. B 80 (2009) 140511.

[60] T. J. Liu, J. Hu, B. Qian, D. Fobes, Z. Q. Mao, W. Bao, M. Reehuis, S. A. J. Kimber, K. Prokes, S. Matas, D. N. Argyriou, A. Hiess, A. Rotaru, H. Pham, L. Spinu, Y. Qiu, V. Thampy, A. T. Savici, J. A. Rodriguez, C. Broholm, From $(\pi, 0)$ magnetic order to superconductivity with $(\pi, \pi)$ magnetic resonance in $\mathrm{Fe}_{1.02}\left(\mathrm{Te}_{1-x} \mathrm{Se}_{x}\right)$, Nature Mater. 9 (2010) 718.

[61] P. L. Paulose, C. S. Yadav, K. M. Subhedar, Magnetic phase diagram of $\mathrm{fe}_{1.1} \mathrm{te}_{1-x} \mathrm{se}_{x}$ : A comparative study with the stoichiometric superconducting fete ${ }_{1-x} \mathrm{se}_{x}$ system, Euro. Phys. Lett. 90 (2010) 27011.

[62] N. Katayama, S. Ji, D. Louca, S.-H. Lee, M. Fujita, T. J. Sato, J. S. Wen, Z. J. Xu, G. D. Gu, G. Xu, Z. W. Lin, M. Enoki, S. Chang, K. Yamada, J. M. Tranquada, Investigation of the spin-glass regime between the antiferromagnetic and superconducting phases in $\mathrm{fe}_{1+y} \mathrm{se}_{x} \mathrm{te}_{1-x}$, J. Phys. Soc. Jpn. 79 (2010) 113702.

[63] Y. Mizuguchi, Y. Takano, Review of fe chalcogenides as the simplest febased superconductor, J. Phys. Soc. Jpn. 79 (2010) 102001.

[64] C. Dong, H. Wang, Z. Li, J. Chen, H. Q. Yuan, M. Fang, Revised phase diagram for the fete ${ }_{1-x} \mathrm{se}_{x}$ system with fewer excess fe atoms Phys. Rev. B 84 (2011) 224506. doi:10.1103/PhysRevB.84.224506. URL http://link . aps .org/doi/10.1103/PhysRevB.84.224506 
[65] J. Dong, H. J. Zhang, G. Xu, Z. Li, G. Li, W. Z. Hu, D. Wu, G. F. Chen, X. Dai, J. L. Luo, Z. Fang, N. L. Wang, Competing orders and spin-density-wave instability in la $\left(\mathrm{o}_{1-x} \mathrm{f}_{x}\right)$ feas, Euro. Phys. Lett. 83 (2008) 27006 .

[66] V. Cvetkovic, Z. Tesanovic, Multiband magnetism and superconductivity in fe-based compounds, Euro. Phys. Lett. 85 (2009) 37002.

[67] S. Graser, T. A. Maier, P. J. Hirschfeld, D. J. Scalapino, Near-degeneracy of several pairing channels in multiorbital models for the fe pnictides, New J. Phys. 11 (2009) 025016.

[68] H. Kotegawa, S. Masaki, Y. Awai, H. Tou, Y. Mizuguchi, Y. Takano, Evidence for unconventional superconductivity in arsenic-free iron-based superconductor fese: A ${ }^{77}$ se-nmr study, J. Phys. Soc. Jpn. 77 (2008) 113703.

[69] S. Onari, H. Kontani, Self-consistent vertex correction analysis for iron-based superconductors: Mechanism of coulomb interactiondriven orbital fluctuations, Phys. Rev. Lett. 109 (2012) 137001. doi:10.1103/PhysRevLett.109.137001 URL http://link.aps.org/doi/10.1103/PhysRevLett.109.137001

[70] H. Kontani, Y. Inoue, T. Saito, Y. Yamakawa, S. Onari, Orbital fluctuation theory in iron-based superconductors: -wave superconductivity, structure transition, and impurity-induced nematic order, Solid State Communications 152 (8) (2012) 718 - 727. doi:10.1016/j.ssc.2012.01.012

[71] G. R. Stewart, Superconductivity in iron compounds, Rev. Mod. Phys. 83 (2011) 1589-1652. doi:10.1103/RevModPhys.83.1589. URL http://link.aps .org/doi/10.1103/RevModPhys.83.1589

[72] M. R. Norman, The challenge of unconventional superconductivity, Science 332 (2011) 196. 
[73] P. J. Hirschfeld, M. M. Korshunov, I. I. Mazin, Gap symmetry and structure of fe-based superconductors, Reports on Progress in Physics 74 (12) (2011) 124508.

[74] F. Wang, D.-H. Lee, The electron-pairing mechanism of iron-based superconductors, Science 332 (2011) 200-204.

[75] J. Wen, G. Xu, G. Gu, J. M. Tranquada, R. J. Birgeneau, Interplay between magnetism and superconductivity in iron-chalcogenide superconductors: crystal growth and characterizations, Rep. Pro. Phys. 74 (12) (2011) 124503.

[76] P. Dai, J. Hu, E. Dagotto, Magnetism and its microscopic origin in ironbased high-temperature superconductors, Nat Phys. 8 (10) (2012) $709-$ 718. doi:10.1038/nphys2438.

[77] K. Ishida, Y. Nakai, H. Hosono, To what extent iron-pnictide new superconductors have been clarified: A progress report, J. Phys. Soc. Jpn. 78 (2009) 062001.

[78] J. W. Lynn, P. Dai, Neutron studies of the iron-based family of high $t_{c}$ magnetic superconductors, Physcia C 469 (2009) 469.

[79] M. D. Lumsden, A. D. Christianson, Magnetism in Fe-based superconductors, J. Phys.: Conden. Matter 22 (2010) 203203.

[80] M. Wu, F. Hsu, K. Yeh, T. Huang, J. Luo, M. Wang, H. Chang, T. Chen, S. Rao, B. Mok, C. Chen, Y. Huang, C. Ke, P. Wu, A. Chang, C. Wu, T. Perng, The development of the superconducting pbo-type $\beta$-fese and related compounds, Physica C 469 (2009) 340.

[81] P. C. Canfield, S. L. Bud'ko, Feas-based superconductivity: A case study of the effects of transition metal doping on $\mathrm{bafe}_{2} \mathrm{as}_{2}$, Annual Rev. Conden. Matter Phys. 1 (2010) 27. 
[82] D. C. Johnston, The puzzle of high temperature superconductivity in layered iron pnictides and chalcogenides, Adv. Phys. 59 (2010) 803.

[83] J. Paglione, R. L. Greene, High-temperature superconductivity in ironbased materials, Nature Phys. 6 (2010) 645.

[84] S. Medvedev, T. M. McQueen, I. Trojan, T. Palasyuk, M. I. Eremets, R. J. Cava, S. Naghavi, F. Casper, V. Ksenofontov, G. Wortmann, C. Felser, Electronic and magnetic phase diagram of $\alpha$-fe $\mathrm{f}_{1.01}$ se with superconductivity at $36.7 \mathrm{k}$ under pressure, Nature Mater. 8 (2009) 630.

[85] S. Margadonna, Y. Takabayashi, Y. Ohishi, Y. Mizuguchi, Y. Takano, T. Kagayama, T. Nakagawa, M. Takata, K. Prassides, Pressure evolution of the low-temperature crystal structure and bonding of the superconductor fese $\left(t_{c}=37 \mathrm{k}\right)$, Phys. Rev. B 80 (2009) 064506.

[86] S. Masaki, H. Kotegawa, Y. Hara, H. Tou, K. Murata, Y. Mizuguchi, Y. Takano, Precise pressure dependence of the superconducting transition temperature of fese: Resistivity and ${ }^{77}$ se-nmr study, J. Phys. Soc. Jpn. 78 (2009) 063704.

[87] Q.-Y. Wang, Z. Li, W.-H. Zhang, Z.-C. Zhang, J.-S. Zhang, W. Li, H. Ding, Y.-B. Ou, P. Deng, K. Chang, J. Wen, C.-L. Song, K. He, J.-F. Jia, S.-H. Ji, Y. Wang, L. Wang, X. Chen, X. Ma, Q.-K. Xue, Interface induced high temperature superconductivity in single unit-cell fese films on srtio3, Chin. Phys. Lett. 29 (2012) 037402.

[88] S. Tan, Y. Zhang, M. Xia, Z. Ye, F. Chen, X. Xie, R. Peng, D. Xu, Q. Fan, H. Xu, J. Jiang, T. Zhang, X. Lai, T. Xiang, J. Hu, B. Xie, D. Feng, Interface-induced superconductivity and strain-dependent spin density waves in fese/srtio3 thin films, Nat Mater 12 (7) (2013) 634-640.

[89] S. He, J. He, W. Zhang, L. Zhao, D. Liu, X. Liu, D. Mou, Y.-B. Ou, Q.-Y. Wang, Z. Li, L. Wang, Y. Peng, Y. Liu, C. Chen, L. Yu, G. Liu, X. Dong, J. Zhang, C. Chen, Z. Xu, X. Chen, X. Ma, Q. Xue, X. J. Zhou, Phase 
diagram and electronic indication of high-temperature superconductivity at 65k in single-layer fese films, Nat Mater 12 (7) (2013) 605-610.

[90] D. Liu, W. Zhang, D. Mou, J. He, Y.-B. Ou, Q.-Y. Wang, Z. Li, L. Wang, L. Zhao, S. He, Y. Peng, X. Liu, C. Chen, L. Yu, G. Liu, X. Dong, J. Zhang, C. Chen, Z. Xu, J. Hu, X. Chen, X. Ma, Q. Xue, X. J. Zhou, Electronic origin of high-temperature superconductivity in single-layer fese superconductor, Nat Commun 3 (2012) 931, 10.1038/ncomms1946.

[91] J.-F. Ge, Z.-L. Liu, C. Liu, C.-L. Gao, D. Qian, Q.-K. Xue, Y. Liu, J.F. Jia, Superconductivity above $100 \mathrm{k}$ in single-layer fese films on doped srtio3, Nat Mater 14 (3) (2015) 285-289.

[92] Y. Sun, W. Zhang, Y. Xing, F. Li, Y. Zhao, Z. Xia, L. Wang, X. Ma, Q.K. Xue, J. Wang, High temperature superconducting fese films on srtio3 substrates, Sci. Rep. 4 (2014) 6040.

[93] I. Bozovic, C. Ahn, A new frontier for superconductivity, Nat Phys 10 (12) (2014) 892-895.

[94] R. Currat, Neutron and X-Ray Spectroscopy, Springer, 2006, Ch. ThreeAxis Inelastic Neutron Scattering.

[95] G. Shirane, S. M. Shapiro, J. M. Tranquada, Neutron Scattering with a Triple-Axis Spectrometer: Basic Techniques, Cambridge University Press, Cambridge, 2002.

[96] G. L. Squires, Introduction to the Theory of Thermal Neutron Scattering, Dover, Mineola, NY, 1996.

[97] I. A. Zaliznyak, S.-H. Lee, Modern Techniques for Characterizing Magnetic Materials, Springer, Heidelberg, 2005, Ch. Magnetic Neutron Scattering.

[98] G. Xu, Z. Xu, J. M. Tranquada, Absolute cross-section normalization of magnetic neutron scattering data, Rev. Sci. Instr. 84 (8) (2013) 083906. 
[99] W. Bao, Y. Qiu, Q. Huang, M. A. Green, P. Zajdel, M. R. Fitzsimmons, M. Zhernenkov, S. Chang, M. Fang, B. Qian, E. K. Vehstedt, J. Yang, H. M. Pham, L. Spinu, Z. Q. Mao, Tunable $(\delta \pi, \delta \pi)$-type antiferromagnetic order in $\alpha$-fe(te,se) superconductors,

Phys. Rev. Lett. $102 \quad$ (2009) 247001. doi:10.1103/PhysRevLett.102.247001 URL http://link.aps.org/doi/10.1103/PhysRevLett.102.247001

[100] S. Li, C. de la Cruz, Q. Huang, Y. Chen, J. W. Lynn, J. Hu, Y.-L. Huang, F.-C. Hsu, K.-W. Yeh, M.-K. Wu, P. Dai, First-order magnetic and structural phase transitions in $\mathrm{fe}_{1+y} \mathrm{se}_{x} \mathrm{te}_{1-x}$, Phys. Rev. B 79 (2009) 054503 .

[101] A. Martinelli, A. Palenzona, M. Tropeano, C. Ferdeghini, M. Putti, M. R. Cimberle, T. D. Nguyen, M. Affronte, C. Ritter, From antiferromagnetism to superconductivity in $\mathrm{fe}_{1+y} \mathrm{te}_{1-x} \mathrm{se}_{x}(0 \leq x \leq 0.20)$ : Neutron powder diffraction analysis, Phys. Rev. B 81 (2010) 094115.

[102] Y. Mizuguchi, F. Tomioka, S. Tsuda, T. Yamaguchi, Y. Takano, Superconductivity in s-substituted fete, Appl. Phys. Lett. 94 (2009) 012503.

[103] Y. Mizuguchi, F. Tomioka, S. Tsuda, T. Yamaguchi, Y. Takano, Fete as a candidate material for new iron-based superconductor, Physica C 469 (2009) 1027.

[104] A. Subedi, L. Zhang, D. J. Singh, M.-H. Du, Density functional study of fes, fese and fete: Electronic structure, magnetism, phonons and superconductivity, Phys. Rev. B 78 (2008) 134514.

[105] Y. Xia, D. Qian, L. Wray, D. Hsieh, G. F. Chen, J. L. Luo, N. L. Wang, M. Z. Hasan, Fermi surface topology and low-lying quasiparticle dynamics of parent fe $e_{1+x}$ te/se superconductor, Phys. Rev. Lett. 103 (2009) 037002.

[106] K. Nakayama, T. Sato, P. Richard, T. Kawahara, Y. Sekiba, T. Qian, G. F. Chen, J. L. Luo, N. L. Wang, H. Ding, T. Takahashi, Angle- 
resolved photoemission spectroscopy of the iron-chalcogenide superconductor $\mathrm{fe}_{1.03} \mathrm{te}_{0.7} \mathrm{Se}_{0.3}$ : Strong coupling behavior and the universality of interband scattering, Phys. Rev. Lett. 105 (2010) 197001.

[107] D. Fruchart, P. Convert, P. Wolfers, R. Madar, J. P. Senateur, R. Fruchart, Structure antiferroma gnetique de $\mathrm{fe}_{1.125}$ te accompagnee d'une deformation monoclinique, Mater. Res. Bull. 10 (1975) 169.

[108] A. V. Balatsky, D. Parker, Not all iron superconductors are the same, Physics 2 (2009) 59.

[109] I. A. Zaliznyak, Z. Xu, J. M. Tranquada, G. Gu, A. M. Tsvelik, M. B. Stone, Unconventional temperature enhanced magnetism in fe $\mathrm{f}_{1.1}$ te, Phys. Rev. Lett. 107 (2011) 216403.

[110] M. J. Han, S. Y. Savrasov, Doping driven $(\pi, 0)$ nesting and magnetic properties of fe $e_{1+x}$ te superconductors, Phys. Rev. Lett. 103 (2009) 067001.

[111] F. Ma, W. Ji, J. Hu, Z.-Y. Lu, T. Xiang, First-principles calculations of the electronic structure of tetragonal $\alpha$-fete and $\alpha$-fese crystals: Evidence for a bicollinear antiferromagnetic order, Phys. Rev. Lett. 102 (2009) 177003.

[112] A. M. Turner, F. Wang, A. Vishwanath, Kinetic magnetism and orbital order in iron telluride, Phys. Rev. B 80 (2009) 224504.

[113] C. Fang, B. A. Bernevig, J. Hu, Theory of magnetic order in fe $e_{1+y} \mathrm{te}_{1-x} \mathrm{se}_{x}$, Euro. Phys. Lett. 86 (2009) 67005.

[114] C.-Y. Moon, H. J. Choi, Chalcogen-height dependent magnetic interactions and magnetic order switching in fese $\mathrm{te}_{1-x}$, Phys. Rev. Lett. 104 (2010) 057003.

[115] W.-G. Yin, C.-C. Lee, W. Ku, Unified picture for magnetic correlations in iron-based superconductors, Phys. Rev. Lett. 105 (2010) 107004. 
[116] D. Parshall, G. Chen, L. Pintschovius, D. Lamago, T. Wolf, L. Radzihovsky, D. Reznik, Cbmpetition between commensurate and incommensurate magnetic ordering in $\mathrm{Fe}_{1+y}$ Te, Phys. Rev. B 85 (2012) 140515. doi:10.1103/PhysRevB.85.140515

URL http://link.aps.org/doi/10.1103/PhysRevB.85.140515

[117] C. Stock, E. E. Rodriguez, M. A. Green, Spin fluctuations and superconductivity in powders of $\mathrm{fe}_{1+x} \mathrm{te}_{0.7} \mathrm{Se}_{0.3}$ as a function of interstitial iron concentration, Phys. Rev. B $85 \quad$ (2012) 094507. doi:10.1103/PhysRevB.85.094507.

URL http://link .aps .org/doi/10.1103/PhysRevB.85.094507

[118] D. Fobes, I. A. Zaliznyak, Z. Xu, R. Zhong, G. Gu, J. M. Tranquada, L. Harriger, D. Singh, V. O. Garlea, M. Lumsden, B. Winn, Ferro-orbital ordering transition in iron telluride $\mathrm{fe}_{1+y}$ te, Phys. Rev. Lett. 112 (2014) 187202. doi:10.1103/PhysRevLett.112.187202. URL http://link.aps.org/doi/10.1103/PhysRevLett.112.187202

[119] X. Liu, C.-C. Lee, Z. J. Xu, J. S. Wen, G. Gu, W. Ku, J. M. Tranquada, J. P. Hill, X-ray diffuse scattering study of local distortions in $\mathrm{Fe}_{1+x} \mathrm{Te}$ induced by excess Fe, Phys. Rev. B 83 (2011) 184523.

[120] V. Thampy, J. Kang, J. A. Rodriguez-Rivera, W. Bao, A. T. Savici, J. Hu, T. J. Liu, B. Qian, D. Fobes, Z. Q. Mao, C. B. Fu, W. C. Chen, Q. Ye, R. W. Erwin, T. R. Gentile, Z. Tesanovic, C. Broholm, Friedel-like oscillations from interstitial iron in superconducting fe $f_{1+y} \mathrm{te}_{0.62} \mathrm{Se}_{0.38}$, $\begin{array}{lllll}\text { Phys. } & \text { Rev. } & \text { Lett. } & 108 & \text { (2012) }\end{array}$ doi:10.1103/PhysRevLett.108.107002 URL http://link.aps.org/doi/10.1103/PhysRevLett.108.107002

[121] L. Zhang, D. J. Singh, M. H. Du, Density functional study of excess fe in $\mathrm{fe}_{1+x}$ te: Magnetism and doping, Phys. Rev. B 79 (2009) 012506.

[122] E. E. Rodriguez, C. Stock, P. Zajdel, K. L. Krycka, C. F. Majkrzak, P. Zavalij, M. A. Green, Magnetic-crystallographic phase diagram of 
the superconducting parent compound fe 1 $_{1+x}$ te, Phys. Rev. B 84 (2011) 064403.

[123] I. A. Zaliznyak, Z. J. Xu, J. S. Wen, J. M. Tranquada, G. D. Gu, V. Solovyov, V. N. Glazkov, A. I. Zheludev, V. O. Garlea, M. B. Stone, Continuous magnetic and structural phase transitions in $\mathrm{fe}_{1+y} \mathrm{te}$, Phys. Rev. B 85 (2012) 085105. doi:10.1103/PhysRevB.85.085105. URL http://link.aps .org/doi/10.1103/PhysRevB.85.085105

[124] Y. Mizuguchi, K. Hamada, K. Goto, H. Takatsu, H. Kadowaki, O. Miura, Evolution of two-step structural phase transition in fe1+dte detected by low-temperature x-ray diffraction, Solid State Commun. 152 (12) (2012) 1047 - 1051. doi:10.1016/j.ssc.2012.03.022. URL http://wWw.sciencedirect.com/science/article/pii/S0038109812001603

[125] S. Ducatman, R. M. Fernandes, N. B. Perkins, Theory of the evolution of magnetic order in $\mathrm{fe}_{1+y}$ te, arXiv:1408.1418.

[126] M. Enayat, Z. Sun, U. R. Singh, R. Aluru, S. Schmaus, A. Yaresko, Y. Liu, C. Lin, V. Tsurkan, A. Loidl, J. Deisenhofer, P. Wahl, Real-space imaging of the atomic-scale magnetic structure of fe1+yte, Science 345 (6197) (2014) 653-656.

[127] Jinsheng Wen, Guangyong Xu, Zhijun Xu, Zhi Wei Lin, Qiang Li, W. Ratcliff, G. Gu, J. M. Tranquada, Short-range incommensurate magnetic order near the superconducting phase boundary in $\mathrm{fe}_{1+\delta} \mathrm{te}_{1-x} \mathrm{se}_{x}$, Phys. Rev. B 80 (2009) 104506.

[128] J. Wen, G. Xu, Z. Xu, Z. W. Lin, Q. Li, Y. Chen, S. Chi, G. Gu, J. M. Tranquada, Effect of magnetic field on the spin resonance in fete $0.5 \mathrm{se}_{0.5}$ as seen via inelastic neutron scattering, Phys. Rev. B 81 (2010) 100513(R).

[129] Z. Xu, J. Wen, G. Xu, Q. Jie, Z. Lin, Q. Li, S. Chi, D. K. Singh, G. Gu, J. M. Tranquada, Disappearance of static magnetic order and evolution of spin fluctuations in $\mathrm{fe}_{1+\delta} \mathrm{se}_{x} \mathrm{te}_{1-x}$, Phys. Rev. B 82 (2010) 104525. 
[130] M. Bendele, P. Babkevich, S. Katrych, S. N. Gvasaliya, E. Pomjakushina, K. Conder, B. Roessli, A. T. Boothroyd, R. Khasanov, H. Keller, Tuning the superconducting and magnetic properties of $\mathrm{fe}_{y} \mathrm{Se}_{0.25} \mathrm{te}_{0.75}$ by varying the iron content, Phys. Rev. B 82 (2010) 212504.

[131] T. M. McQueen, A. J. Williams, P. W. Stephens, J. Tao, Y. Zhu, V. Ksenofontov, F. Casper, C. Felser, R. J. Cava, Tetragonal-to-orthorhombic structural phase transition at $90 \mathrm{k}$ in the superconductor $\mathrm{fe}_{1.01} \mathrm{se}$, Phys. Rev. Lett. 103 (2009) 057002.

[132] S. Margadonna, Y. Takabayashi, M. T. McDonald, K. Kasperkiewicz, Y. Mizuguchi, Y. Takano, A. N. Fitch, E. Suard, K. Prassides, Crystal structure of the new fese ${ }_{1-x}$ superconductor, Chem. Commun. (2008) 5607 .

[133] E. Pomjakushina, K. Conder, V. Pomjakushin, M. Bendele, R. Khasanov, Synthesis, crystal structure, and chemical stability of the superconductor fese $_{1-x}$, Phys. Rev. B 80 (2009) 024517.

[134] T. M. McQueen, Q. Huang, V. Ksenofontov, C. Felser, Q. Xu, H. Zandbergen, Y. S. Hor, J. Allred, A. J. Williams, D. Qu, J. Checkelsky, N. P. Ong, R. J. Cava, Extreme sensitivity of superconductivity to stoichiometry in fe $_{1+\delta}$ se, Phys. Rev. B 79 (2009) 014522.

[135] T. Imai, K. Ahilan, F. L. Ning, T. M. McQueen, R. J. Cava, Why does undoped fese become a high $t_{c}$ superconductor under pressure?, Phys. Rev. Lett. 102 (2009) 177005.

[136] M. Bendele, A. Amato, K. Conder, M. Elender, H. Keller, H.-H. Klauss, H. Luetkens, E. Pomjakushina, A. Raselli, R. Khasanov, Pressure induced static magnetic order in superconducting fese ${ }_{1-x}$, Phys. Rev. Lett. 104 (2010) 087003.

[137] H.-Y. Cao, S. Tan, H. Xiang, D. L. Feng, X.-G. Gong, Interfacial effects on the spin density wave in fese/ $/$ srtio $_{3}$ thin films, 
Phys. Rev. B 89 (2014) 014501. doi:10.1103/PhysRevB.89.014501.

URL http://link . aps .org/doi/10.1103/PhysRevB.89.014501

[138] H.-Y. Cao, S. Chen, H. Xiang, X.-G. Gong, An insulating ground state with novel antiferromagnetic order in fese monolayer, arXiv:1407.7145.

[139] O. J. Lipscombe, G. F. Chen, C. Fang, T. G. Perring, D. L. Abernathy, A. D. Christianson, T. Egami, N. Wang, J. Hu, P. Dai, Spin waves in the $(\pi, 0)$ magnetically ordered iron chalcogenide fe $e_{1.05}$ te, Phys. Rev. Lett. 106 (2011) 057004.

[140] M. D. Lumsden, A. D. Christianson, E. A. Goremychkin, S. E. Nagler, H. A. Mook, M. B. Stone, D. L. Abernathy, T. Guidi, G. J. MacDougall, C. De La Cruz, A. S. Sefat, M. A. McGuire, B. C. Sales, D. Mandrus, Evolution of spin excitations into the superconducting state in fete ${ }_{1-x} \mathrm{Se}_{x}$, Nature Phys. 6 (2010) 182.

[141] S.-H. Lee, G. Xu, W. Ku, J. S. Wen, C. C. Lee, N. Katayama, Z. J. Xu, S. Ji, Z. W. Lin, G. D. Gu, H.-B. Yang, P. D. Johnson, Z.-H. Pan, T. Valla, M. Fujita, T. J. Sato, S. Chang, K. Yamada, J. M. Tranquada, Coupling of spin and orbital excitations in the iron-based superconductor fete $_{0.5} \mathrm{se}_{0.5}$, Phys. Rev. B 81 (2010) 220502(R).

[142] Z. Xu, J. Wen, G. Xu, S. Chi, W. Ku, G. Gu, J. M. Tranquada, Localmoment magnetism in superconducting fete $\mathrm{e}_{0.35} \mathrm{Se}_{0.65}$ as seen via inelastic neutron scattering, Phys. Rev. B 84 (2011) 052506.

[143] Z. Xu, J. Wen, Y. Zhao, M. Matsuda, W. Ku, X. Liu, G. Gu, D.-H. Lee, R. J. Birgeneau, J. M. Tranquada, G. Xu, Temperature-dependent transformation of the magnetic excitation spectrum on approaching superconductivity in $\mathrm{fe}_{1+y-x}(\mathrm{Ni} / \mathrm{Cu})_{x} \mathrm{te}_{0.5} \mathrm{se}_{0.5}$, Phys. Rev. Lett. 109 (2012) 227002. doi:10.1103/PhysRevLett.109.227002. URL http://link.aps.org/doi/10.1103/PhysRevLett.109.227002 
[144] S. Li, C. Zhang, M. Wang, H.-q. Luo, X. Lu, E. Faulhaber, A. Schneidewind, P. Link, J. Hu, T. Xiang, P. Dai, Normal-state hourglass dispersion of the spin excitations in fese $\mathrm{fe}_{x-x}$, Phys. Rev. Lett. 105 (2010) 157002.

[145] D. N. Argyriou, A. Hiess, A. Akbari, I. Eremin, M. M. Korshunov, J. Hu, B. Qian, Z. Mao, Y. Qiu, C. Broholm, W. Bao, Incommensurate itinerant antiferromagnetic excitations and spin resonance in the fete ${ }_{0.6} \mathrm{se}_{0.4}$ superconductor, Phys. Rev. B 81 (2010) 220503.

[146] P. Babkevich, M. Bendele, A. T. Boothroyd, K. Conder, S. N. Gvasaliya, R. Khasanov, E. Pomjakushina, B. Roessli, Magnetic excitations of $\mathrm{fe}_{1+y} \mathrm{Se}_{x} \mathrm{te}_{1-x}$ in magnetic and superconductive phases, J. Phys. Conden. Matter 22 (2010) 142202.

[147] Z. Xu, J. Wen, J. Schneeloch, A. D. Christianson, R. J. Birgeneau, G. Gu, J. M. Tranquada, G. Xu, Low-energy magnetic excitations from the $\mathrm{fe}_{1+y-z}(\mathrm{ni} / \mathrm{cu})_{z} \mathrm{te}_{1-x} \mathrm{Se}_{x}$ system, Phys. Rev. B 89 (2014) 174517. doi:10.1103/PhysRevB.89.174517. URL http://link. aps.org/doi/10.1103/PhysRevB.89.174517

[148] H. Zhai, F. Wang, D.-H. Lee, Ahtiferromagnetically driven electronic correlations in iron pnictides and cuprates, Phys. Rev. B 80 (2009) 064517. doi:10.1103/PhysRevB.80.064517. URL http://link.aps.org/doi/10.1103/PhysRevB.80.064517

[149] R. M. Fernandes, A. V. Chubukov, J. Knolle, I. Eremin, J. Schmalian, Preemptive nematic order, pseudogap, and orbital order in the iron pnictides, Phys. Rev. B 85 (2012) 024534. doi:10.1103/PhysRevB.85.024534. URL http://link. aps.org/doi/10.1103/PhysRevB.85.024534

[150] H. Ikeda, R. Arita, J. Kuneš, Phase diagram and gap anisotropy in iron-pnictide superconductors, Phys. Rev. B 81 (5) (2010) 054502. doi:10.1103/PhysRevB.81.054502 
[151] A. V. Chubukov, M. G. Vavilov, A. B. Vorontsov, Momentum dependence and nodes of the superconducting gap in the iron pnictides,

Phys. Rev. B 80 (2009) 140515. doi:10.1103/PhysRevB.80.140515.

URL http://link .aps .org/doi/10.1103/PhysRevB.80.140515

[152] W.-C. Lee, W. Lv, J. M. Tranquada, P. W. Phillips, Impact of dynamic orbital correlations on magnetic excitations in the normal state of iron-based superconductors, Phys. Rev. B 86 (2012) 094516. doi:10.1103/PhysRevB.86.094516 URL http://link.aps.org/doi/10.1103/PhysRevB.86.094516

[153] G. Yu, Y. Li, E. M. Motoyama, M. Greven, A universal relationship between magnetic resonance and superconducting gap in unconventional superconductors, Nature Phys. 5 (2009) 873.

[154] T. A. Maier, D. J. Scalapino, Theory of neutron scattering as a probe of the superconducting gap in the iron pnictides, Phys. Rev. B 78 (2008) 020514(R).

[155] T. A. Maier, S. Graser, D. J. Scalapino, P. Hirschfeld, Neutron scattering resonance and the iron-pnictide superconducting gap, Phys. Rev. B 79 (2009) 134520.

[156] E. Demler, S.-C. Zhang, Theory of the resonant neutron scattering of high- $t_{c}$ superconductors, Phys. Rev. Lett. 75 (1995) 4126.

[157] C. D. Batista, G. Ortiz, A. V. Balatsky, Unified description of the resonance peak and incommensuration in high- $t_{c}$ superconductors, Phys. Rev. B 64 (2001) 172508.

[158] A. D. Christianson, E. A. Goremychkin, R. Osborn, S. Rosenkranz, M. D. Lumsden, C. D. Malliakas, I S. Todorov, H. Claus, D. Y. Chung, M. G. Kanatzidis, R. I. Bewley, T. Guidi, Unconventional superconductivity in $\mathrm{ba}_{0.6} \mathrm{k}_{0} .4 \mathrm{fe}_{2} \mathrm{as}_{2}$ from inelastic neutron scattering, Nature 456 (2008) 930. 
[159] S.-i. Shamoto, M. Ishikado, A. D. Christianson, M. D. Lumsden, S. Wakimoto, K. Kodama, A. Iyo, M. Arai, Inelastic neutron scattering study of the resonance mode in the optimally doped pnictide superconductor lafeaso $_{0.92} \mathrm{f}_{0.08}$, Phys. Rev. B 82 (2010) 172508.

[160] I. I. Mazin, J. Schmalian, Pairing symmetry and pairing state in ferropnictides: Theoretical overview, Physica C 469 (2009) 614.

[161] Y. Qiu, W. Bao, Y. Zhao, C. Broholm, V. Stanev, Z. Tesanovic, Y. C. Gasparovic, S. Chang, J. Hu, B. Qian, M. Fang, Z. Mao, Spin gap and resonance at the nesting wave vector in superconducting fese $e_{0.4} \mathrm{te}_{0.6}$, Phys. Rev. Lett. 103 (2009) 067008.

[162] H. A. Mook, M. D. Lumsden, A. D. Christianson, S. E. Nagler, B. C. Sales, R. Jin, M. A. McGuire, A. S. Sefat, D. Mandrus, T. Egami, C. dela Cruz, Unusual relationship between magnetism and superconductivity in fete $_{0.5} \mathrm{se}_{0.5}$, Phys. Rev. Lett. 104 (2010) 187002.

[163] P. Dai, H. A. Mook, G. Aeppli, S. M. Hayden, F. Doğan, J. Yu, Y. Yanagida, H. Takashima, Y. Inaguma, M. Itoh, T. Nakamura, Resonance as a measure of pairing correlations in the high- $t_{c}$ superconductor ybco, Nature 406 (2000) 965.

[164] J. M. Tranquada, C. H. Lee, K. Yamada, Y. S. Lee, L. P. Regnault, H. M. Rønnow, Evidence for an incommensurate magnetic resonance in $\mathrm{la}_{2-x} \mathrm{Sr}_{x} \mathrm{CuO}_{4}$, Phys. Rev. B 69 (2004) 174507.

[165] J. Zhao, L.-P. Regnault, C. Zhang, M. Wang, Z. Li, F. Zhou, Z. Zhao, C. Fang, J. Hu, P. Dai, Neutron spin resonance as a probe of the superconducting energy gap of bafe ${ }_{1.9} \mathrm{ni}_{0.1}$ as $_{2}$ superconductors, Phys. Rev. B 81 (2010) 180505.

[166] P. Bourges, B. Keimer, S. Pailhòes, L. Regnault, Y. Sidis, C. Ulrich, The resonant magnetic mode: A common feature of high-tc superconductors, Physica C 424 (2005) 45. 
[167] W. Bao, A. T. Savici, G. E. Granroth, C. Broholm, K. Habicht, Y. Qiu, J. Hu, T. Liu, Z. Q. Mao, A Triplet Resonance in Superconducting $\mathrm{FeSe}_{0.4} \mathrm{Te}_{0.6}$, arXiv:1002.1617.

[168] S. Li, X. Lu, M. Wang, H.-q. Luo, M. Wang, C. Zhang, E. Faulhaber, L.-P. Regnault, D. Singh, P. Dai, Effect of the in-plane magnetic field on the neutron spin resonance in optimally doped fese ${ }_{0.4} \mathrm{te}_{0.6}$ and bafe $\mathrm{b}_{1.9} \mathrm{ni}_{0.1} \mathrm{as}_{2}$ superconductors, Phys. Rev. B 84 (2011) 024518.

[169] C. Stock, C. Broholm, Y. Zhao, F. Demmel, H. J. Kang, K. C. Rule, C. Petrovic, Magnetic field splitting of the spin resonance in cecoin 5 Phys. Rev. Lett. 109 (2012) 167207. doi:10.1103/PhysRevLett.109.167207. URL http://link.aps.org/doi/10.1103/PhysRevLett.109.167207

[170] S. Raymond, J. Panarin, G. Lapertot, J. Flouquet, Evolution of the spin resonance of cecoin $_{5}$ as a function of magnetic field and La substitution, Journal of the Physical Society of Japan 80SB (Supplement B) (2011) SB023. doi:10.1143/JPSJS.80SB.SB023.

URL http://jpsj . ipap.jp/link?JPSJS/80SB/SB023/

[171] J. Panarin, S. Raymond, G. Lapertot, J. Flouquet, Evolution of the spin resonance in cecoin $_{5}$ under magnetic field,

Journal of the Physical Society of Japan 78 (11) (2009) 113706. doi:10.1143/JPSJ.78.113706.

URL http://jpsj . ipap.jp/link?JPSJ/78/113706/

[172] C. Stock, E. E. Rodriguez, M. A. Green, P. Zavalij, J. A. RodriguezRivera, Interstitial iron tuning of the spin fluctuations in the nonsuperconducting parent phase fe ${ }_{1+x}$ te, Phys. Rev. B 84 (2011) 045124.

[173] G. Xu, G. D. Gu, M. Hücker, B. Fauque, T. G. Perring, L. P. Regnault, J. M. Tranquada, Testing the itinerancy of spin dynamics in superconducting $\mathrm{bi}_{2} \mathrm{Sr}_{2} \mathrm{Cacu}_{2} \mathrm{O}_{8+\delta}$, Nature Phys. 5 (2009) 642. 
[174] R. Klingeler, N. Leps, I. Hellmann, A. Popa, U. Stockert, C. Hess, V. Kataev, H.-J. Grafe, F. Hammerath, G. Lang, S. Wurmehl, G. Behr, L. Harnagea, S. Singh, B. Büchner, Local antiferromagnetic correlations in the iron pnictide superconductors lafeaso $_{1-x} f_{x}$ and $c a\left(f e_{1-x} \mathrm{Co}_{x}\right)_{2} a s_{2}$ as seen via normal-state susceptibility, Phys. Rev. B 81 (2010) 024506.

[175] S. Chadov, D. Schärf, G. H. Fecher, C. Felser, L. Zhang, D. J. Singh, Electronic structure, localization, and spin-state transition in cu-substituted fese:fe ${ }_{1-x} \mathrm{cu}_{x}$ se, Phys. Rev. B 81 (2010) 104523.

[176] D. J. Singh, M.-H. Du, Density functional study of lafeaso ${ }_{1-x} f_{x}$ : A low carrier density superconductor near itinerant magnetism, Phys. Rev. Lett. 100 (2008) 237003.

[177] S. O. Diallo, V. P. Antropov, T. G. Perring, C. Broholm, J. J. Pulikkotil, N. Ni, S. L. Bud'ko, P. C. Canfield, A. Kreyssig, A. I. Goldman, R. J. McQueeney, Itinerant magnetic excitations in antiferromagnetic $\operatorname{cafe}_{2} \mathrm{as}_{2}$, Phys. Rev. Lett. 102 (2009) 187206.

[178] Z. P. Yin, S. Lebègue, M. J. Han, B. P. Neal, S. Y. Savrasov, W. E. Pickett, Electron-hole symmetry and magnetic coupling in antiferromagnetic lafeaso, Phys. Rev. Lett. 101 (2008) 047001.

[179] K. Matan, R. Morinaga, K. Iida, T. J. Sato, Anisotropic itinerant magnetism and spin fluctuations in bafe[sub 2] as[sub 2]: A neutron scattering study, Phys. Rev. B 79 (2009) 054526.

[180] L. Ke, M. van Schilfgaarde, J. Pulikkotil, T. Kotani, V. Antropov, Low-energy coherent stoner-like excitations in cafe $_{2} \mathrm{as}_{2}$, Phys. Rev. B 83 (2011) 060404. doi:10.1103/PhysRevB.83.060404. URL http://link .aps .org/doi/10.1103/PhysRevB.83.060404

[181] J. Wu, P. Phillips, A. H. Castro Neto, Theory of the magnetic moment in iron pnictides, Phys. Rev. Lett. 101 (2008) 126401. 
[182] M. Liu, L. W. Harriger, H. Luo, M. Wang, R. A. Ewings, T. Guidi, H. Park, K. Haule, G. Kotliar, S. M. Hayden, P. Dai, Nature of magnetic excitations in superconducting bafe ${ }_{1.9} \mathrm{ni}_{0.1} \mathrm{as}_{2}$, Nat Phys. 8 (5) (2012) 376381. doi:10.1038/nphys2268

[183] Z. Tesanovic, Are iron pnictides new cuprates?, Physics 2 (2009) 60.

[184] M. M. Qazilbash, J. J. Hamlin, R. E. Baumbach, L. Zhang, D. J. Singh, M. B. Maple, D. N. Basov, Electronic correlations in the iron pnictides, Nature Phys. 5 (2009) 647.

[185] H. Gretarsson, A. Lupascu, J. Kim, D. Casa, T. Gog, W. Wu, S. R. Julian, Z. J. Xu, J. S. Wen, G. D. Gu, R. H. Yuan, Z. G. Chen, N.-L. Wang, S. Khim, K. H. Kim, M. Ishikado, I. Jarrige, S. Shamoto, J.-H. Chu, I. R. Fisher, Y.-J. Kim, Revealing the dual nature of magnetism in iron pnictides and iron chalcogenides using x-ray emission spectroscopy, Phys. Rev. B 84 (2011) 100509. doi:10.1103/PhysRevB.84.100509. URL http://link.aps.org/doi/10.1103/PhysRevB.84.100509

[186] R. Hu, E. S. Bozin, J. B. Warren, C. Petrovic, Superconductivity, magnetism, and stoichiometry of single crystals of $\mathrm{fe}_{1+y}\left(\mathrm{te}_{1-x} \mathrm{~s}_{x}\right)_{z}$, Phys. Rev. B 80 (2009) 214514.

[187] P. C. Canfield, S. L. Bud'ko, N. Ni, J. Q. Yan, A. Kracher, Decoupling of the superconducting and magnetic/structural phase transitions in electron-doped bafe ass $_{2}$, Phys. Rev. B 80 (2009) 060501 (R).

[188] S. Ideta, T. Yoshida, I. Nishi, A. Fujimori, Y. Kotani, K. Ono, Y. Nakashima, S. Yamaichi, T. Sasagawa, M. Nakajima, K. Kihou, Y. Tomioka, C. H. Lee, A. Iyo, H. Eisaki, T. Ito, S. Uchida, R. Arita, Dependence of carrier doping on the impurity potential in transitionmetal-substituted feas-based superconductors, Phys. Rev. Lett. 110 (2013)

107007. doi:10.1103/PhysRevLett.110.107007.

URL http://link.aps.org/doi/10.1103/PhysRevLett.110.107007 
[189] G. Levy, R. Sutarto, D. Chevrier, T. Regier, R. Blyth, J. Geck, S. Wurmehl, L. Harnagea, H. Wadati, T. Mizokawa, I. S. Elfimov, A. Damascelli, G. A. Sawatzky, Probing the role of co substitution in the electronic structure of iron pnictides,

$\begin{array}{lllll}\text { Phys. } & \text { Rev. } & \text { Lett. } & 109 & \text { (2012) }\end{array}$ doi:10.1103/PhysRevLett.109.077001 URL http://link.aps.org/doi/10.1103/PhysRevLett.109.077001

[190] C. Liu, A. D. Palczewski, R. S. Dhaka, T. Kondo, R. M. Fernandes, E. D. Mun, H. Hodovanets, A. N. Thaler, J. Schmalian, S. L. Bud'ko, P. C. Canfield, A. Kaminski, Importance of the fermi-surface topology to the superconducting state of the electron-doped pnictide ba(fe $\left.\mathrm{fe}_{1-x} \mathrm{CO}_{x}\right)_{2} \mathrm{as}_{2}$, Phys. Rev. B 84 (2) (2011) 020509. doi:10.1103/PhysRevB.84.020509.

[191] N. Ni, A. Thaler, J. Q. Yan, A. Kracher, E. Colombier, S. L. Bud'ko, P. C. Canfield, S. T. Hannahs, Temperature versus doping phase diagrams for $\mathrm{Ba}\left(\mathrm{Fe}_{1-x} T M_{x}\right)_{2} \mathrm{as}_{2}(T M=$ ni, cu, cu/co) single crystals, Phys. Rev. B 82 (2010) 024519. doi:10.1103/PhysRevB.82.024519.

URL http://link. aps .org/doi/10.1103/PhysRevB.82.024519

[192] M. Neupane, P. Richard, Y.-M. Xu, K. Nakayama, T. Sato, T. Takahashi, A. V. Federov, G. Xu, X. Dai, Z. Fang, Z. Wang, G.-F. Chen, N.-L. Wang, H.-H. Wen, H. Ding, Electron-hole asymmetry in the superconductivity of doped $\mathrm{BaFe}_{2} \mathrm{As}_{2}$ seen via the rigid chemical-potential shift in photoemission, Phys. Rev. B 83 (2011) 094522. doi:10.1103/PhysRevB. 83.094522 URL http://link.aps.org/doi/10.1103/PhysRevB.83.094522

[193] K. Nakamura, R. Arita, H. Ikeda, Frrst-principles calculation of transition-metal impurities in LaFeAsO, Phys. Rev. B 83 (2011) 144512. doi:10.1103/PhysRevB.83.144512

URL http://link.aps.org/doi/10.1103/PhysRevB.83.144512

[194] S. Konbu, K. Nakamura, H. Ikeda, R. Arita, Fermi-suface evolution by transition-metal substitution in the iron-based superconductor 
LaFeAsO, Journal of the Physical Society of Japan 80 (12) (2011) 123701.

doi:10.1143/JPSJ.80.123701.

URL http://jpsj.ipap.jp/link?JPSJ/80/123701/

[195] H. Wadati, I. Elfimov, G. A. Sawatzky, Where are the extra $d$ electrons in transition-metal-substituted iron pnictides?,

Phys. Rev. Lett. $105 \quad$ (2010) 157004.

doi:10.1103/PhysRevLett.105.157004

URL http://link.aps.org/doi/10.1103/PhysRevLett.105.157004

[196] T. Berlijn, C.-H. Lin, W. Garber, W. Ku, Do transition-metal substitutions dope carriers in iron-based superconductors?,

$\begin{array}{lllll}\text { Phys. } & \text { Rev. } & \text { Lett. } & 108 & \text { (2012) }\end{array}$

doi:10.1103/PhysRevLett.108.207003

URL http://link.aps .org/doi/10.1103/PhysRevLett.108.207003

[197] E. M. Bittar, C. Adriano, T. M. Garitezi, P. F. S. Rosa, L. Mendon ça Ferreira, F. Garcia, G. d. M. Azevedo, P. G. Pagliuso, E. Granado, Cbsubstitution effects on the fe valence in the $\mathrm{BaFe}_{2} \mathrm{As}_{2}$ superconducting compound: A study of hard x-ray absorption spectroscopy, Phys. Rev. Lett. 107 (2011) 267402. doi:10.1103/PhysRevLett.107.267402. URL http://link.aps.org/doi/10.1103/PhysRevLett.107.267402

[198] H.-H. Kuo, J.-H. Chu, S. C. Riggs, L. Yu, P. L. McMahon, K. De Greve, Y. Yamamoto, J. G. Analytis, I. R. Fisher, Plossible origin of the nonmonotonic doping dependence of the in-plane resistivity anisotropy of $\mathrm{Ba}\left(\mathrm{Fe}_{1-x} T_{x}\right)_{2} \mathrm{As}_{2}(T=\mathrm{Co}, \mathrm{Ni}$ and $\mathrm{Cu})$, Phys. Rev. B 84 (2011) 054540. doi:10.1103/PhysRevB.84.054540

URL http://link.aps.org/doi/10.1103/PhysRevB.84.054540

[199] J. A. McLeod, A. Buling, R. J. Green, T. D. Boyko, N. A. Skorikov, E. Z. Kurmaev, M. Neumann, L. D. Finkelstein, N. Ni, A. Thaler, S. L. Bud'ko, P. C. Canfield, A. Moewes, Effect of 3d doping on the electronic structure of bafe $a_{2} a_{2}$ Journal 
of Physics: Condensed Matter 24 (21) (2012) 215501.

URL http://stacks . iop.org/0953-8984/24/i=21/a=215501

[200] E. L. Thomas, W. Wong-Ng, D. Phelan, J. N. Millican, Thermopower of co-doped fese, J. Appl. Phys. 105 (2009) 073906.

[201] A. J. Williams, T. M. McQueen, V. Ksenofontov, C. Felser, R. J. Cava, The metal-insulator transition in $\mathrm{fe}_{1.01-x} \mathrm{cu}_{x} \mathrm{se}, \mathrm{J}$. Phys. Condens. Matter 21 (2009) 305701.

[202] T.-W. Huang, T.-K. Chen, K.-W. Yeh, C.-T. Ke, C. L. Chen, Y.-L. Huang, F.-C. Hsu, M.-K. Wu, P. M. Wu, M. Avdeev, A. J. Studer, Doping-driven structural phase transition and loss of superconductivity in $m_{x} \mathrm{fe}_{1-x} \mathrm{se}_{\delta}$ ( $m=\mathrm{mn}, \mathrm{cu})$, Phys. Rev. B 82 (2010) 104502.

[203] Y. Mizuguchi, F. Tomioka, S. Tsuda, T. Yamaguchi, Y. Takano, Substitution effects on fese superconductor, J. Phys. Soc. Jpn. 78 (2009) 074712.

[204] D. J. Gawryluk, J. Fink-Finowicki, A. Wisniewski, R. Puzniak, V. Domukhovski, R. Diduszko, M. Kozlowski, M. Berkowski, Growth conditions, structure, and superconductivity of pure and metal-doped $\mathrm{FeTe}_{1-x} \mathrm{Se}_{x}$ single crystals, Supercond. Sci. Tech. 24 (2011) 065011.

[205] R. Shipra, H. Takeya, K. Hirata, A. Sundaresan, Effects of ni and co doping on the physical properties of tetragonal fese $\mathrm{e}_{0.5} \mathrm{te}_{0.5}$ superconductor, Physica C 470 (2010) 528.

[206] S. B. Zhang, H. Lei, X. Zhu, G. Li, B. Wang, L. Li, X. Zhu, W. Song, Z. Yang, Y. Sun, Divergency of sdw and structure transition in fe $\mathrm{fe}_{1-x} \mathrm{ni}_{x} \mathrm{se}_{0.82}$ superconductors, Physica C 469 (2009) 1958.

[207] J. Wen, S. Li, Z. Xu, C. Zhang, M. Matsuda, O. Sobolev, J. T. Park, A. D. Christianson, E. Bourret-Courchesne, Q. Li, G. Gu, D.-H. Lee, J. M. Tranquada, G. Xu, R. J. Birgeneau, Enhanced low-energy magnetic excitations via suppression of the itinerancy in $\mathrm{Fe}_{0.98-z} \mathrm{Cu}_{z} \mathrm{Te}_{0.5} \mathrm{Se}_{0.5}$, Phys. Rev. B 
88 (2013) 144509. doi:10.1103/PhysRevB.88.144509.

URL http://link.aps .org/doi/10.1103/PhysRevB.88.144509

[208] J. Wang, R. Zhong, S. Li, Y. Gan, Z. Xu, C. Zhang, T. Ozaki, M. Matsuda, Y. Zhao, Q. Li, G. Xu, G. Gu, J. M. Tranquada, R. J. Birgeneau, J. Wen, Substitution of $\mathrm{Ni}$ for $\mathrm{Fe}$ in superconducting $\mathrm{Fe}_{0.98} \mathrm{Te}_{0.5} \mathrm{Se}_{0.5}$ depresses the normal-state conductivity but not the magnetic spectral weight, Phys. Rev. B 91 (2015) 014501. doi:10.1103/PhysRevB.91.014501. URL http://link.aps.org/doi/10.1103/PhysRevB.91.014501

[209] J. Wen, Z. Xu, G. Xu, M. D. Lumsden, P. N. Valdivia, E. BourretCourchesne, G. Gu, D.-H. Lee, J. M. Tranquada, R. J. Birgeneau, Magnetic order tuned by cu substitution in $\mathrm{fe}_{1.1-z} \mathrm{cu}_{z}$ te, Phys. Rev. B 86 (2012) 024401. doi:10.1103/PhysRevB.86.024401. URL http://link.aps.org/doi/10.1103/PhysRevB.86.024401

[210] B. Joseph, A. Iadecola, A. Puri, L. Simonelli, Y. Mizuguchi, Y. Takano, N. L. Saini, Evidence of local structural inhomogeneity in fese ${ }_{1-x} \mathrm{te}_{x}$ from extended x-ray absorption fine structure, Phys. Rev. B 82 (2010) $020502(\mathrm{R})$.

[211] H. Hu, J.-M. Zuo, J. Wen, Z. Xu, Z. Lin, Q. Li, G. Gu, W. K. Park, L. H. Greene, Phase separation in the iron chalcogenide superconductor $\mathrm{fe}_{1+y} \mathrm{te}_{x} \mathrm{se}_{1-x}$, New J. Phys. 13 (2011) 053031.

[212] X. He, G. Li, J. Zhang, A. B. Karki, R. Jin, B. C. Sales, A. S. Sefat, M. A. McGuire, D. Mandrus, E. W. Plummer, Nanoscale chemical phase separation in fete f.55 $_{0.45} \mathrm{Se}_{0.45}$ as seen via scanning tunneling spectroscopy, Phys. Rev. B 83 (2011) 220502.

[213] T. Taen, Y. Tsuchiya, Y. Nakajima, T. Tamegai, Superconductivity at $t_{c} \sim 14 \mathrm{k}$ in single-crystalline fete ${ }_{0.61} \mathrm{se}_{0.39}$, Phys. Rev. B 80 (2009) 092502.

[214] T. Noji, T. Suzuki, H. Abe, T. Adachi, M. Kato, Y. Koike, Growth, annealing effects on superconducting and magnetic properties, and 
anisotropy of fese ${ }_{1-x} \mathrm{te}_{x}(0.5 \leq x \leq 1)$ single crystals, J. Phys. Soc. Jpn. 79 (2010) 084711.

[215] K. W. Yeh, C. T. Ke, T. W. Huang, T. K. Chen, Y. L. Huang, P. M. Wu, M. K. Wu, Superconducting $\mathrm{FeSe}_{1-x} \mathrm{Te}_{x}$ Single Crystals Grown by Optical Zone-Melting Technique, Cryst. Growth Des. 9 (2009) 4847.

[216] Y. Mizuguchi, K. Deguchi, Y. Kawasaki, T. Ozaki, M. Nagao, S. Tsuda, T. Yamaguchi, Y. Takano, Superconductivity in oxygenannealed $\mathrm{FeTe}_{1-x} \mathrm{~S}_{x}$ single crystal, J. Appl. Phys. 109 (2011) 013914.

[217] R. Hu, H. Lei, M. Abeykoon, E. S. Bozin, S. J. L. Billinge, J. B. Warren, T. Siegrist, C. Petrovic, Synthesis, crystal structure, and magnetism of $\beta$-fe $\mathrm{e}_{1.00(2)} \mathrm{Se}_{1.00(3)}$ single crystals, Phys. Rev. B 83 (2011) 224502.

[218] K. K. Huynh, Y. Tanabe, T. Urata, H. Oguro, S. Heguri, K. Watanabe, K. Tanigaki, Electric transport of a single-crystal iron chalcogenide fese superconductor: Evidence of symmetry-breakdown nematicity and additional ultrafast dirac cone-like carriers, Phys. Rev. B 90 (2014) 144516. doi:10.1103/PhysRevB.90.144516.

URL http://link . aps .org/doi/10.1103/PhysRevB.90.144516 